\title{
Adhäsivbrücken als Therapieoptionen zum minimalinvasiven Lückenschluss
}

Andreas Zenthöfer, Cornelia Frese, Wolfgang Bömicke

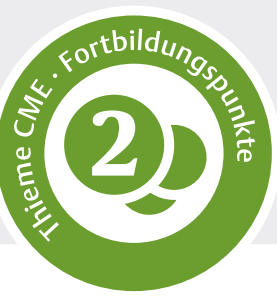

In diesem Artikel werden Adhäsivbrücken als minimalinvasive Therapieoptionen zur Versorgung von Einzelzahnlücken aus dem Spektrum der Zahnerhaltung sowie der zahnärztlichen Prothetik gegenübergestellt und Indikationsbereiche aufgezeigt. Die genannten Vorgehensweisen geben Einblick in die vielfältigen Einsatzgebiete der Adhäsivbrücken und ermöglichen es, patientenzentrierte Versorgungskonzepte zum Lückenschluss in der Praxis anzubieten.

\section{Einleitung}

Einzelzahnlücken können in verschiedenen Lebensabschnitten vorliegen. Nicht angelegte Zähne oder Traumata spielen vorwiegend bei Jugendlichen oder jüngeren Erwachsenen eine Rolle. Zahnverluste aufgrund starker Zerstörung, nicht lösbarer endodontischer Probleme oder fortschreitender Parodontalerkrankungen betreffen hingegen eher ältere Menschen. Grundsätzlich stehen für die Therapie von Einzelzahnlücken im Front- und Seitenzahnbereich verschiedene etablierte Konzepte zur Verfügung [1]. In der Regel müssen bei einer patientenzentrierten Entscheidungsfindung für eine Behandlungsstrategie mehrere Faktoren berücksichtigt und gegeneinander abgewogen werden. Hierzu zählen bspw. die Präferenzen des Patienten, das Nutzen-Risiko-Verhältnis unter Beachtung individueller prognostischer Faktoren, Invasivität und anatomischen Gegebenheiten sowie das Nutzen-Kosten-Verhältnis [2,3]. Klassischerweise werden Einzelzahnlücken durch konventionelle Brücken oder implantatverankerte Einzelkronen geschlossen. Beide Versorgungsformen sind in der zahnmedizinischen Literatur gut untersucht und zeigen eine vergleichbare klinische Leistungsfähigkeit [1].

\section{Laborgefertigte Adhäsivbrücken}

Sind die Nachbarzähne (weitestgehend) karies- und füllungsfrei und kommt eine Implantatlösung aufgrund individueller Restriktionen nicht in Frage [4-6], gilt es auch abzuwägen, ob anstelle einer klassischen Brückenpräparation mit Entfernung von bis zu rund 70 Massenprozent [7,8] der klinischen Kronen der Pfeilerzähne nicht weniger invasivem Zahnersatz der Vorzug gegeben werden kann. In den 1970er-Jahren wurde eine Therapieoption vorgestellt, einzelne fehlende Zähne mit an den Nachbarzähnen verklebten Nichtedelmetallgerüsten zu ersetzen $[9,10]$ : die Adhäsivbrücke (auch Klebebrücke oder Maryland-Brücke genannt). Allerdings führte erst die Ergänzung der Technik um ein geschlossenes Adhäsivflügeldesign (frühe Modelle besaßen perforierte Retainer zur makromechanischen Verankerung des Befestigungszements) mit Mikroretentionen [11] und ein retentives Präparationsdesign [12] dazu, dass diese Versorgungsform auch als definitiver Zahnersatz dienen konnte. Weiterentwicklungen der zahn- und restaurationsseitigen Haftvermittlung sowie weitere Modifikationen des retentiven Präparationsdesigns in den 1980er-2000erJahren sorgen heute dafür, dass Adhäsivbrücken bei adäquater Indikationsstellung eine mit konventionellem Zahnersatz vergleichbare Therapieoption darstellen [13-16]. Eine aktuelle Übersichtsarbeit schlägt vor, Adhäsivbrücken aufgrund ihrer guten klinischen Leistungsfähigkeit viel häufiger als definitive Versorgung in Erwägung zu ziehen, als dies bislang der Fall ist [16]. Insbesondere komplexe Behandlungssituationen, bei denen verschiedene Fachdisziplinen involviert sind (z. B. Lücken bei Spaltpatienten, Zahntraumata oder Nichtanlagen bei Patienten unter 18 Jahren) stellen eine potenzielle Indikation für die minimalinvasive Therapie mittels Adhäsivbrücken dar. Ein zwischenzeitlicher Rückschritt hinsichtlich der Überlebensraten (v. a. durch Frakturen, aber auch Retentionsverluste) kam u. a. dadurch zustande, dass zunehmend vollkeramische Adhäsivbrücken hergestellt wurden, um dem Patientenwunsch nach metallfreien, hochästhetischen Versorgungen gerecht zu werden [17, 18]. In den letzten Jahren gab es aber auch hier zahlreiche und deutliche Optimierungen $[3,19,20]$.

\section{Faserverstärkte Kompositbrücken}

Als ebenso minimalinvasive und gleichzeitig frugale Therapieoption [21,22], besteht alternativ die Möglichkeit, (temporäre) Brücken aus faserverstärkten Kompositen (FRC) zu fertigen. Faserverstärkte Komposite werden seit längerer Zeit in technischen Bereichen wie Schiffbau, Luftfahrt, Fahrzeugbau und bei der Erzeugung von Windenergie eingesetzt $[23,24]$. Bereits Anfang der 1960er- 
Jahre wurden FRCs in der Zahntechnik verwendet, um Prothesenbasen zu verstärken [25]. Aus werkstoffkundlicher Sicht bestand der Nachteil der FRC-Materialien jedoch im zeitlich limitierten chemischen Verbund zwischen Fasern und der Methacrylat-basierten Kompositmatrix. Im 1. Schritt der Entwicklung war eine erfolgreiche chemische Verbindung von Faser- und Komposittechnologie notwendig, um die mechanischen Defizite konventioneller Methacrylat-basierter Kompositmaterialien wie Polymerisationsschrumpfung, Frakturresistenz und Sprödigkeit auszugleichen und einen möglichst dauerhaften klinischen Einsatz in der Zahnheilkunde zu ermöglichen [26]. Für die klinische Anwendung stehen dem Behandler heute verschiedene Arten von FRC-Materialien zur Verfügung: FRC-Stränge mit parallel angeordneten Fasern zur Herstellung von FRC-Brücken, FRC-Netze zur internen Verstärkung von direkten Restaurationen oder Bulk-Fill-Materialien mit kurzen zufällig angeordneten Glasfasern [26].

Aufgrund der oben beschriebenen werkstoffkundlichen Eigenschaften sind materialspezifische Limitationen bis heute ein Schwachpunkt von FRC-Restaurationen. Mit Blick auf den Einzelzahnersatz durch eine 1- oder 2-flügelige FRC-Brücke kann gemäß einem Konsensusworkshop aus dem Jahre 2016 lediglich von einer mittleren Überlebenszeit der FRC-Brücke von ca. 6 Jahren ausgegangen werden [27]. Im Spektrum der Adhäsivbrücken sind die FRC-Brücken somit als semipermanente Versorgung zu betrachten $[28,29]$. Sie weisen jedoch Vorteile auf, die den Einsatz dieser Versorgungsart in gewissen Fällen vorteilhaft machen und rechtfertigen. Besonders hervorzuheben ist hier das „Dynamic Treatment Concept“, welches ein defensives Vorgehen und den maximalen Schutz von Zahnhartsubstanz vor allem bei Kindern und jungen Erwachsenen beinhaltet [27].

Der nachfolgende Artikel soll einen praxisorientierten und evidenzbasierten Überblick über minimalinvasive Therapieoptionen zum Lückenschluss im Front- und Seitenzahnbereich im Rahmen eines patientenzentrierten Vorgehens geben.

\section{Indikationsstellung}

Obwohl sich die Indikationsbereiche von laborgefertigten Adhäsivbrücken und faserverstärkten Kompositbrücken überschneiden, bestehen dennoch Unterschiede in der Indikationsstellung, die im Folgenden aufgezeigt werden. - Abb. 1 zeigt verschiedene minimalinvasive Therapieoptionen.

\section{Adhäsivbrücken}

In Frage kommen Adhäsivbrücken, wenn Lücken im Front- oder Seitenzahnbereich geschlossen werden sollen und keine Stellungs- oder Formkorrekturen an den Nachbarzähnen vorgenommen werden müssen. Die Zähne sollten weitestgehend karies- bzw. füllungsfrei sein, damit ausreichend natürlicher Zahnschmelz für den Haftverbund zwischen Restaurationsmaterial und Zahn zur Verfügung steht. Die Pfeilerzähne sollten dabei keinen oder einen nur geringgradigen Lockerungsgrad ( $L G \leq 1$ ) aufweisen. Adhäsivbrücken können die Therapieoption der 1. Wahl sein, wenn Zahnlücken bei nicht abgeschlossenem Kieferwachstum behandelt werden müssen. Soll die Versorgung später wieder entfernt werden, können präparierte Bereiche recht einfach mit Kompositrestaurationen wiederhergestellt werden. Einflügelige Adhäsiv-

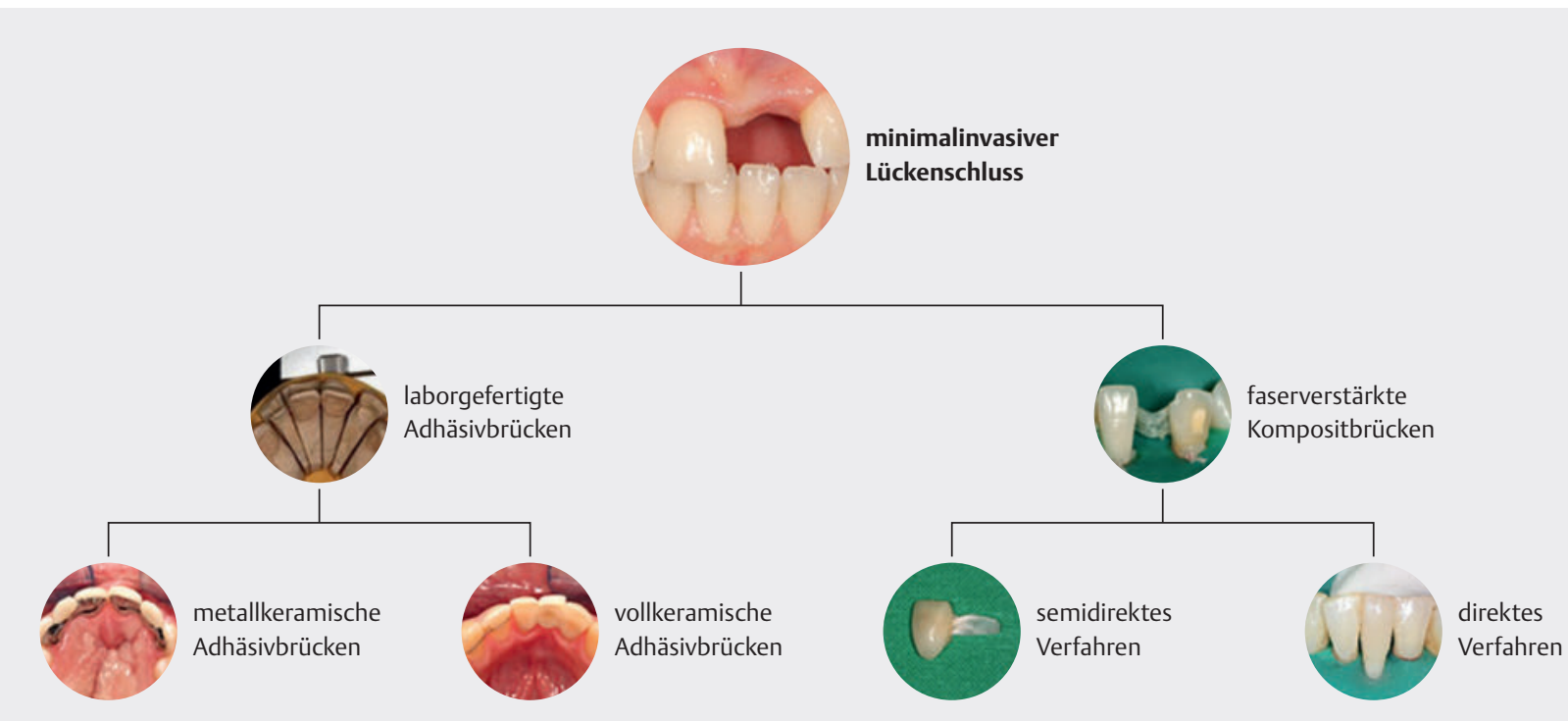

• Abb. 1 Übersicht über verschiedene Therapieoptionen zum minimalinvasiven Ersatz von fehlenden Zähnen. 
brücken können bereits ab dem 10. Lebensjahr eingegliedert werden, wohingegen für die primäre Verblockung mit einer 2-flügeligen Adhäsivbrücke das transversale Kieferwachstum abgeschlossen sein muss, was mit dem Durchbruch der bleibenden Eckzähne (in der Regel ab dem 12. Lebensjahr) der Fall ist.

Ein unbemerktes einseitiges Lösen der Versorgung (partielles Debonding) ist bei 1-flügeligen Adhäsivbrücken ausgeschlossen. Auf diese Weise kann Karies am entsprechenden Pfeilerzahn vermieden werden. Die Restauration kann zudem häufig nach Säuberung erneut eingesetzt werden. Umgekehrt besteht eine gewisse Verschluckungsgefahr, wenn sich die 1-flügelige Adhäsivbrücke spontan löst. Bei der 1-flügeligen Variante sind dynamische Okklusionskontakte im Bereich des Brückenglieds zu vermeiden. Allgemein gilt, dass Patienten mit Parafunktionen wie Knirschen und Pressen (Bruxismus) nicht mit Adhäsivbrücken versorgt werden sollten. Seit 2016 erhalten Patienten - auch nach Vollendung des 21. Lebensjahrs - einen Festkostenzuschuss für 1- oder 2-flügelige Adhäsivbrücken, sofern diese metallbasiert und im Frontzahnbereich vorgesehen sind. Damit die Adhäsivbrücke wirklich minimalinvasiv ausgeführt werden kann, sollte man bedenken, dass nur Werkstoffe mit einem hohen E-Modul wie Cobalt-Chrom-Legierungen oder Oxidkeramiken zur Anwendung kommen können. Nicht zuletzt können einem defektorientierten Vorgehen folgend Adhäsivflügel und Vollkronen zur Verankerung von Brücken kombiniert werden, ohne dass sich dies nachteilig auf die Überlebenswahrscheinlichkeit der Restauration auswirkt [30]. Beispielhaft zeigt $>$ Abb. 2 eine solche Versorgung. In der Zusatzinfo-Box ist das Indikationsspektrum für Adhäsivbrücken zusammengefasst.

\section{ZUSATZINFO}

\section{Indikationen für Adhäsivbrücken}

- Alter: nicht abgeschlossenes Kieferwachstum

- (weitgehende) Reversibilität der Restauration erwünscht

- weitgehend karies- und füllungsfreie Pfeilerzähne

- keine Stellungs- und Formveränderung der Pfeilerzähne erforderlich

- Implantate nicht oder nur mit großem prächirurgischem Aufwand möglich oder nicht gewollt

- ästhetisch hohe Ansprüche (ggf. vollkeramische Adhäsivbrücke)

- parodontal gesunde und feste Pfeilerzähne (Lockerungsgrad $\leq 1$ )

- kein Bruxismus

- ökonomische Aspekte

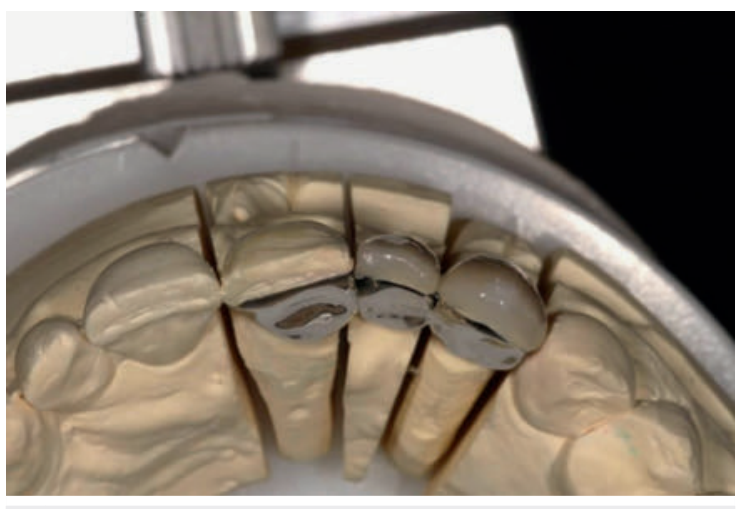

- Abb. 2 Hybrid-Adhäsivbrücke zum Ersatz des seitlichen Schneidezahnes mit Vollkronenanker am Eckzahn und Adhäsivflügel am mittleren Schneidezahn.

\section{Faserverstärkte Kompositbrücken}

FRC-Brücken können im bleibenden Gebiss in jedem Alter als 1- oder 2-flügelige Variante eingesetzt werden. Bei Kindern in der Wachstumsphase sollten 1-flügelige Varianten bevorzugt werden (s.o.). Basierend auf der langjährigen Erfahrung in der klinischen Anwendung vollkeramischer Adhäsivbrücken [19] wird angenommen, dass ein 1-flügeliges Design auch für FRC-Brücken im Frontzahnbereich vorteilhaft für das Überleben ist. Eigene Untersuchungen haben gezeigt, dass FRC-Brücken im Frontzahnbereich eine funktionelle Überlebensrate von 73,5\% aufweisen [29]. In dieser Überlebensrate sind allerdings auch bereits reparierte und noch in situ befindliche FRC-Brücken eingeschlossen. Betrachtet man die Überlebenszeit bis zum 1. Auftreten einer Komplikation (Gesamtüberlebensrate), dann zeigen FRC-Brücken nach 4 Jahren eine Rate von 53,0\% [29].

Besonders hervorzuheben ist bei der Entscheidung für eine FRC-Brücke, dass Pfeilerzähne bereits restaurativ versorgt sein können. Je nach Okklusionsverhältnis ist eine Rillen- oder Kastenpräparation zur Aufnahme des FRC-Flügels indiziert. Falls es die okklusalen Verhältnisse im Frontzahnbereich erlauben, kann der FRC-Strang palatinal/lingual ohne vorherige Präparation aufgelegt werden. Dies ermöglicht nach Entfernung der FRC-Brücke eine Rückkehr zum Status idem, also dem Befund, der vor der Eingliederung der FRC-Brücke bestand [31]. Vor allem im jungen Alter der Patienten ist die Rückkehr zum Status idem ein nicht unerheblicher Vorteil, da der Restaurationszyklus des Zahnes hierdurch wahrscheinlich verzögert wird. Aufgrund der materialspezifischen Limitationen ist das Auftreten von Komplikationen an der FRC-Brücke nicht auszuschließen. Die häufigsten Komplikationen sind Chippingfrakturen, Debonding oder ein kompletter Verlust der FRC-Brücke. Die Defekte können entsprechend den aktuellen Kriterien zur Anfertigung einer intraoralen Reparatur mit Komposit repariert werden und die FRC-Brücke verbleibt in situ. Bei wiederhol- 


ZUSATZINFO
Indikationen für FRC-Brücken
- (nicht) abgeschlossenes Kieferwachstum
- Restitutio ad status idem erwünscht
- Pfeilerzähne können auch bereits restaurativ versorgt
sein
- Möglichkeit der intraoralen Reparatur erwünscht
- Implantate nicht oder nur mit großem prächirur-
gischem Aufwand möglich oder nicht gewollt
- Nichtanlagen bei Kindern und Jugendlichen
- semipermanente Versorgung vor/während einer
Implantation
- Wiederbefestigung eines Echtzahnes nach Extraktion
- kein Bruxismus
- ökonomische Aspekte

ten Misserfolgen liegen möglicherweise eine bisher nicht bekannte Parafunktion oder ungünstige Bissverhältnisse vor und in diesem Fall besteht, analog zu laborgefertigten Adhäsivbrücken, eine Kontraindikation für eine FRC-Brücke. Die Inhalte der Zusatzinfo-Box fassen das Indikationsspektrum für FRC-Brücken zusammen, Unterschiede zur laborgefertigten Adhäsivbrücke sind kursiv dargestellt.

\section{Merke \\ Soll minimalinvasiver Zahnersatz zum Lückenschluss zur Anwendung kommen, ist es wichtig, das Indika- tionsspektrum der jeweiligen Therapieoption ein- zuhalten. Während FRC-Brücken als semipermante Lösung zum Ersatz einzelner Zähne in Frage kommen, können retentive Adhäsivbrücken eine dauerhafte Alternative zu konventionellen Brücken sein.}

\section{Metallkeramische Adhäsivbrücken}

Es ist sinnvoll, im Vorfeld eine Alginatabformung des zu versorgenden Kieferareals zu nehmen, um laborseits eine Tiefziehschiene über ein Set-up (Brückenglied) anfertigen zu lassen. Die Tiefziehschiene kann dann einfach zur Provisoriumherstellung nach Präparation verwendet werden. Des Weiteren dient das Gipsmodell zur Planung der Einschubrichtung und der Ausdehnung der Adhäsivflügel sowie der optimalen Positionierung und Orientierung der Retentionselemente ( $\triangleright$ Abb. $\mathbf{3}$ ). Auch die okklusalen Platzverhältnisse lassen sich beurteilen. Auf eine Anästhesie kann aufgrund der Minimalinvasivität der Präparation verzichtet werden; in den meisten Fällen ist sie aber dennoch vor allem in Hinblick auf die nachfolgende Abformung (Fadenlegen, Blutstillung) hilfreich und sinnvoll.

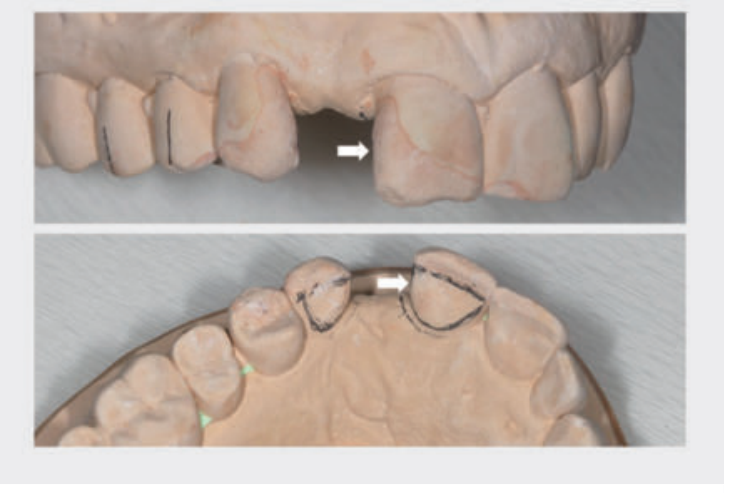

- Abb. 3 Planung der Ausdehnung der Adhäsivflügel: Es wird eine maximale Extension des Adhäsivflügels angestrebt (Ziel ist eine Adhäsivflügelfläche von mindestens $30 \mathrm{~mm}^{2}$ ). Dabei ist jedoch darauf zu achten, dass die Adhäsivflügelränder nicht in den sichtbaren Bereich hineinreichen (weiße Pfeile). Außerdem wird ein Abstand von ca. $2 \mathrm{~mm}$ zur Schneidekante eingehalten, um deren Transluzenz nicht zu kompromittieren.

\section{Präparationsdesign und Behandlungsablauf}

Im 1. Schritt bietet es sich an, an den Pfeilerzähnen die geplante Ausdehnung der Adhäsivflügel mit einem gut kontrastierenden (schwarzen oder blauen) Filzmaler zu markieren. Dies erlaubt nicht nur, die Grenzen der Präparation im Vorfeld zu visualisieren, sondern hilft auch, die sehr diskrete Präparation besser zu kontrollieren ( Abb. 4).

Zunächst wird eine feine Hohlkehlpräparation (Rotringdiamant) der oralen Zahnflächen angelegt und die im Einschub liegenden Unterschnitte werden entfernt. Die Präparation wird dabei so weit wie möglich in den Approximalraum extendiert, um eine maximale Klebefläche zu erreichen. Richtung Zahnlücke muss dabei darauf geachtet werden, dass hierdurch später möglichst keine unverblendeten Metallareale im Bereich der Konnektoren des Zwischenglieds entstehen. Anschließend wird das Tuberculum mit einem kugel- oder eiförmigen Diamanten präpariert. Hierbei gilt es zweierlei zu beachten: der Abschluss der Präparation nach inzisal sollte je nach Transluzenz 1-2 mm apikal der Schneidekante liegen, um ein späteres Durchschimmern der Adhäsivflügel zu verhindern bzw. zu minimieren. Sind Kontaktpunkte im Bereich des Tuberculums vorhanden, muss zudem darauf geachtet werden, mindestens 0,5-0,7 mm Substanz abzutragen, um eine ausreichende Stärke des Adhäsivflügels zu ermöglichen. Statische Okklusionskontakte müssen so geplant werden, dass diese entweder auf dem oder außerhalb des Adhäsivflügels liegen. Kontakte auf der Klebefuge dagegen sind zu vermeiden. Besteht auch ohne Präparation bereits ein ausreichendes vertikales Platzangebot, um eine adäquate Flügeldicke zu gewährleisten (z. B. in der Unterkieferfront oder in der Oberkieferfront 


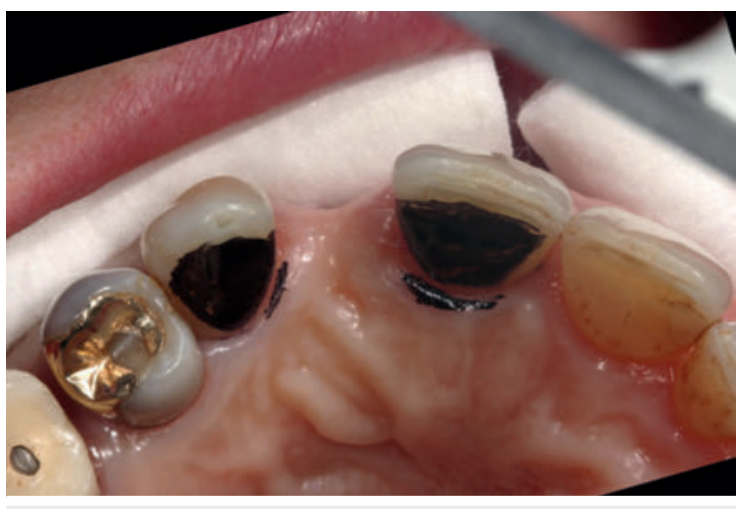

- Abb. 4 Die geplante Ausdehnung des Adhäsivflügels wurde mittels schwarzen Filzmalers an den Pfeilerzähnen markiert. Die Markierung hilft u. a. dabei, die diskrete Präparation sichtbar zu machen.

bei einer entsprechend ausgeprägten sagittalen Stufe), muss für eine effektive Klebung dennoch darauf geachtet werden, dass der gesamte vom Adhäsivflügel bedeckte Schmelzbereich zumindest angeraut wurde. Auch hier hilft die Filzmaler-Markierung die Vollständigkeit der Präparation sicherzustellen. Weiterhin wird pro Pfeilerzahn jeweils eine vertikale Rille zum späteren Brückenglied hin angelegt, welche $\mathrm{min} .3 \mathrm{~mm}$, besser $4 \mathrm{~mm}$ lang sein sollte. Diese kann auch den Abschluss der Präparation nach vestibulär bilden. Die retentive Wirkung der Rille kann unmittelbar mit einer Parodontalsonde ausgetestet werden (die Parodontalsonde lässt sich innerhalb der Rille nicht verkippen bzw. die Sonde rutscht beim Verkippen in orovestibulärer Richtung nicht aus der Rille). Die Präparation wird abschließend durch eine ca. $1 \mathrm{~mm}$ tiefe (gemessen an der oralen Wand der Bohrung) Pinbohrung mittig im Bereich des Tuberculums vervollständigt ( $\bullet$ Abb. 5 ).

Prinzipiell ist es wichtig, dass alle präparierten Retentionselemente einen gemeinsamen Einschub aufweisen, damit der Zahntechniker diese bei der Herstellung der Restauration nicht ausblocken muss und somit deren Funktion eingeschränkt bzw. nicht mehr vorhanden wäre. Ein Kontrollmodell via Alginatteilabformung, die mit schnellhärtendem Gips im Praxislabor ausgegossen wird, oder die Verwendung einer intraoralen Apparatur zur Parallelführung des Winkelstücks (z. B. Parallel-a-Prep; Fa. Dentatus), wie sie auch bei der Präparation für die Aufnahme von Adhäsivattachments zur Anwendung kommt, kann dabei hilfreich sein.

Nun kann die Präzisionsabformung erfolgen. Liegen Präparationsgrenzen para- oder teilweise leicht subgingival, empfiehlt es sich, zu deren besseren Darstellung Fäden zu legen. Die eigentliche Abformung kann bevorzugt einzeitig mit einem dünnfließenden Silikon oder Polyether, mit dem die Pfeiler umspritzt werden, und dem korrespondierenden Löffelmaterial in der Konsistenz knetbar, schwer oder mittelfließend erfolgen. Weitere Behandlungsmaßnahmen können analog zur Herstellung von konventionellem Zahnersatz vorgenommen werden (Alginatabformung des Gegenkiefers, Bissregistrierung usw.). Liegt eine Tiefziehschiene (s. o.) vor, kann ein direktes Provisorium gefertigt werden; dieses sollte mittels provisorischen, eugenolfreien Zements eingegliedert werden und bietet neben der Vermeidung von Zahnstellungsveränderungen auch die Möglichkeit, die Retentivität der Gesamtpräparation auszutesten. Soll die Brücke bereits sehr zeitnah eingesetzt werden und existiert keine Vorabformung, können die präparierten Areale alter-

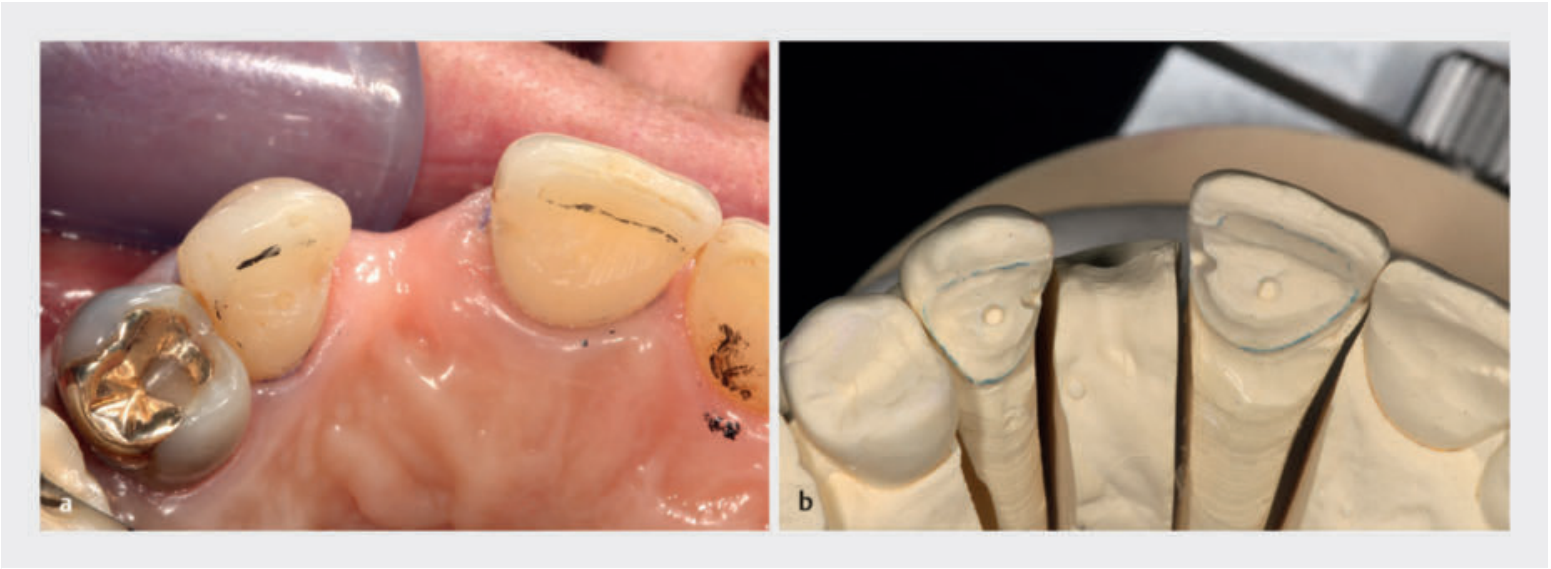

- Abb. 5 Fertige Präparation. Eine definierte umlaufende Präparationsgrenze wurde geschaffen, Rillen und Bohrungen angelegt und Unterschnitte im durch die Orientierung der Rillen und Bohrungen determinierten Einschub entfernt. Die palatinale Substanzreduktion orientierte sich an der Mindestschichtdicke des Adhäsivflügels; bei ausreichendem Abstand zum Antagonisten wurde der Schmelz nur angeraut.

a Klinische Ansicht.

b Gipsmodell. 


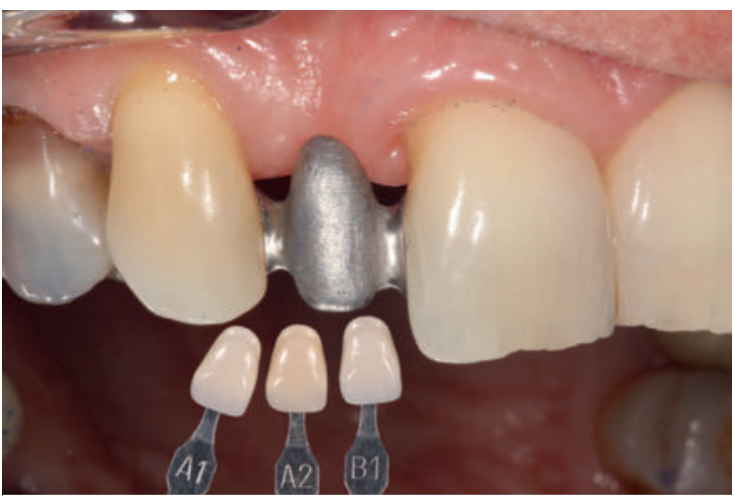

Abb. 6 Die Farbbestimmung erfolgt mit eingesetztem Gerüst.

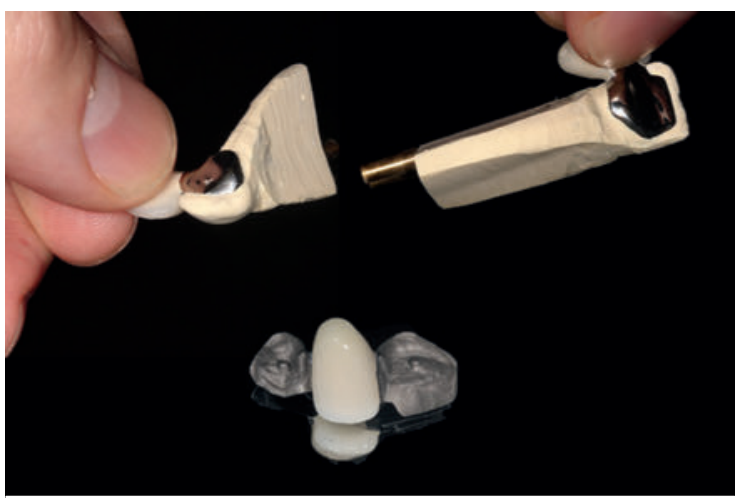

Abb. 7 Überprüfung der Retentivität separat für jeden Pfeilerzahn am Gipsmodell.

nativ mit einem lichthärtenden Kompositmaterial zur provisorischen Versorgung von z. B. Inlay- oder Teilkronenkavitäten abgedeckt werden. Laborseitig kann nun die Adhäsivbrücke im Guss- oder CAD/CAM-Verfahren hergestellt werden. Dem Gussverfahren wird im Zusammenhang mit dem dargestellten Präparationsdesign in der Regel der Vorzug gegeben, da zumindest die Frästechnik bei der exakten Umsetzung der grazilen retentiven Elemente (Bohrungen, Rillen) systembedingt limitiert ist.

Bei der anschließenden Gerüstanprobe werden die Passung (s.u.) und Retentivität sowie die Okklusion der Adhäsivflügel überprüft, außerdem findet die Farbbestimmung statt ( $>$ Abb. 6). Die Farbe erst im Rahmen der Gerüstanprobe zu nehmen, hat dabei den Vorteil, dass ein eventueller Einfluss des Adhäsivflügels auf die Optik des Pfeilerzahnes bei der Verblendung des Zwischenglieds noch berücksichtigt werden kann.

Am Gipsmodell wird die Retentivität für jeden Adhäsivflügel einzeln geprüft. Lässt sich mittels der Brücke der Sägeschnitt orthogonal zur Einschubrichtung halten, dann

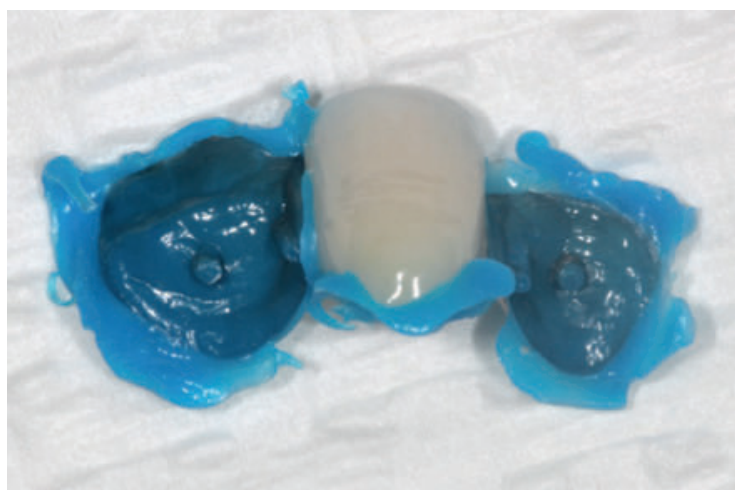

- Abb. 8 Passungskontrolle mittels Fließsilikon. Das Silikon ist nicht durchgedrückt und gleichmäßig dünn, was einer guten Passung entspricht.

kann von einer sehr guten Retentivität ausgegangen werden ( $\vee$ Abb. 7). Klinisch wird die Retentivität bestimmt, indem das Brückenglied von vestibulär belastet wird. Dabei soll idealerweise kein Spalt zwischen dem Pfeilerzahn und dem Adhäsivflügel aufgehen.

Nach dem Verblenden wird die fertige Brücke erneut anprobiert. Zunächst wird die provisorische Versorgung entfernt und die Pfeilerzähne werden mit Sonde, Zirkonium-Silikat-Paste oder Bimsmehl und Polierbürstchen gereinigt. Wenn keine Approximalkontakte im Bereich der Restauration (absolute Mehrzahl der Fälle) liegen, wird zunächst die Innenpassung der Restauration mittels Silikon- oder Polyethermaterial zur Passungskontrolle (z.B. Xantopren oder Fit Checker) überprüft ( $\bullet$ Abb. 8).

Gegebenfalls durchgedrückte Stellen werden markiert und anschließend mittels rotierender Hartmetallinstrumente entfernt. Ist der Materialfilm gleichmäßig dünn und im Bereich der Präparationsgrenze(n) nahezu durchgedrückt, werden die Randpassung mit der zahnärztlichen Sonde kontrolliert sowie die Brücke auf spannungs- und schaukelfreien Sitz hin überprüft. Die Anlagerung des Brückenglieds an das Tegument kann ebenfalls mittels Silikon-/Polyetherprobe oder mit Zahnseide/Superfloss kontrolliert werden. Abschließend wird die Okklusion überprüft. Statische Okklusionskontakte im Bereich der Restaurationsränder bzw. dynamische Okklusionskontakte im Bereich des Brückenglieds sind zu vermeiden und ggf. zu entfernen.

\section{Definitive Eingliederung}

War die Einprobe erfolgreich und sind alle eingeschliffenen Flächen wieder auf Hochglanz poliert (Silikonpolierer: „Greenie, Brownie“; Praxislabor: Poliermotor Ziegenhaar-(Baumwollbürstchen), kann die Adhäsivbrücke eingegliedert werden. Wann immer möglich, sollte hierfür Kofferdam angelegt werden [32]. Ansonsten sollten zumindest Fäden gelegt werden, um ein Aufsteigen von 
- Tab. 1 Ablauf verschiedener adhäsiver Befestigungsstrategien (restaurationsseitig).

\section{Strategie 1}

- Korundstrahlen mit 2-3 bar Druck und einer Partikelgröße von 50 oder $110 \mu \mathrm{m}$

- Reinigung im Ultraschallbad mit Ethanol

- MPD-haltiger, chemisch oder dual härtender Adhäsivzement

\section{Strategie 2}

- Korundstrahlen mit 2-3 bar Druck und einer Partikelgröße von 50 oder $110 \mu \mathrm{m}$

- Reinigung im Ultraschallbad mit Ethano

- MDP-haltiger Primer

- beliebiger, chemisch oder dual härtender Adhäsivzement

\section{Strategie 3}

- tribochemisches Silikatisieren

- Auftragen eines Silans

- beliebiger, chemisch oder dual härtender Adhäsivzement
Sulcusfluid bzw. ein Eindringen des Adhäsivzements in den Sulcus zu vermeiden. Restaurationsseitig wird die Klebefläche mittels Korundstrahlen (Aluminiumoxid; Druck 2-3 bar; Partikelgröße 50-110 $\mu \mathrm{m}$ ) konditioniert. Der Strahlprozess reinigt, aktiviert und vergrößert die Oberfläche bzw. schafft Mikroretentionen zur mechanischen Verankerung des Adhäsivzements. Zum adhäsiven Einsetzen von Nichtedelmetalllegierungen können entweder direkt MDP-haltige Adhäsivzemente (z.B. Panavia 21) verwendet werden oder ein MDP-haltiger Metallprimer (z. B. Alloy Primer) aufgetragen und im Anschluss ein beliebiger Kunststoffzement verwendet werden. Darüber hinaus hat sich auch das tribochemische Silikatisieren der Oberfläche in Kombination mit der Anwendung eines Silans bewährt. > Tab. 1 fasst die restaurationsseitigen Konditionierungsstrategien zusammen.

Im gezeigten Fall wurde die Restauration mittels eines selbstätzenden, auf MDP basierenden Adhäsivsystems (Panavia 21) eingesetzt. Zahnseitig wurden hierfür Schmelzanteile für $10 \mathrm{~s}$ mit $37 \%$ iger Phosphorsäure selektiv geätzt und nach dem Abspülen und Trocken der Klebefläche der selbstätzende, MDP-haltige, rein chemisch härtende Primer auf Dentin und Schmelz aufgetragen. Im Anschluss wurde die Restauration dünn mit dem MDP-haltigen Adhäsivzement bestrichen, eingesetzt und Überschüsse unmittelbar mit Schaumstoffpellets versäubert. Nachdem ein Gel zur Sauerstoffinhibition auf die Restaurationsränder aufgetragen wurde (der Zement härtet rein chemisch unter Sauerstoffausschluss aus), muss die Restauration bis zur vollständigen Polymerisation des Befestigungszements in situ gehalten werden ( Abb. 9).

Die vom Hersteller ausgewiesenen Einwirk- und Polymerisationszeiten der restaurations- und zahnseitig angewendeten Primer und Adhäsive müssen im Rahmen der Konditionierung bzw. Zementierung zwingend eingehalten werden, um einen dauerhaften Verbund zwischen Restauration und Zahn zu garantieren. Abschließend können die Ränder ggf. mit Hartmetallfinierern und für Metall geeigneten Silikonpolierern („Brownie, Greenie“) noch einmal nachgearbeitet werden. $>$ Abb. 10 zeigt die eingegliederte Brücke in situ.

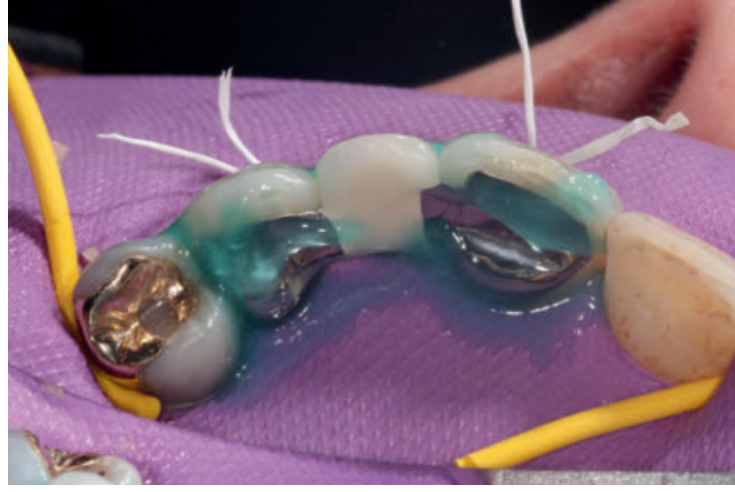

- Abb. 9 Brücke unter Kofferdam zementiert nach Ablauf der Polymerisationszeit des adhäsiven Einsetzzements. Auf die Ränder der Restauration wurde ein Gel (Oxyguard) zum Schutz der Klebefuge vor einem Zutritt von Sauerstoff appliziert. Außerdem im Gel enthalten ist ein Polymerisationsinitiator für den Adhäsivzement.

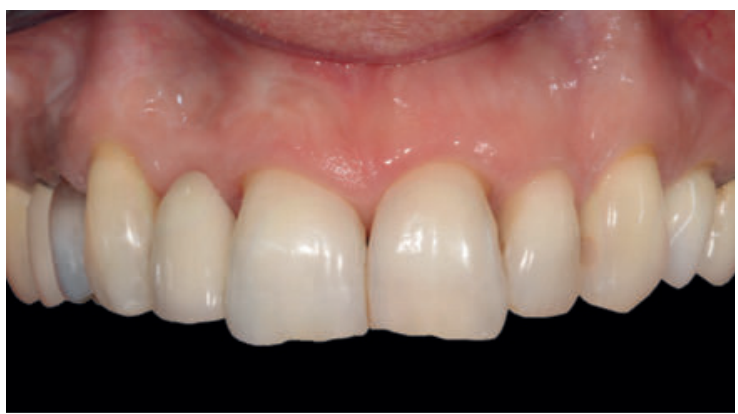

- Abb. 10 Frontalansicht mit eingesetzter Adhäsivbrücke zum minimalinvasiven Ersatz des Zahnes 12.

Der Behandlungsablauf einer 1-flügeligen metallkeramischen Adhäsivbrücke verläuft analog zu dem der hier vorgestellten 2-flügeligen Variante, mit dem Unterschied, dass eine 2. Rille im an den Nachbarzahn grenzenden Approximalbereich angelegt wird, um eine eindeutige Positionierbarkeit (ermöglicht eine Anprobe und das Einsetzen der Restauration ohne zusätzlichen Positionierungsschlüssel) und Retentivität der Restauration sicherzustellen (॰ Abb. 11). 

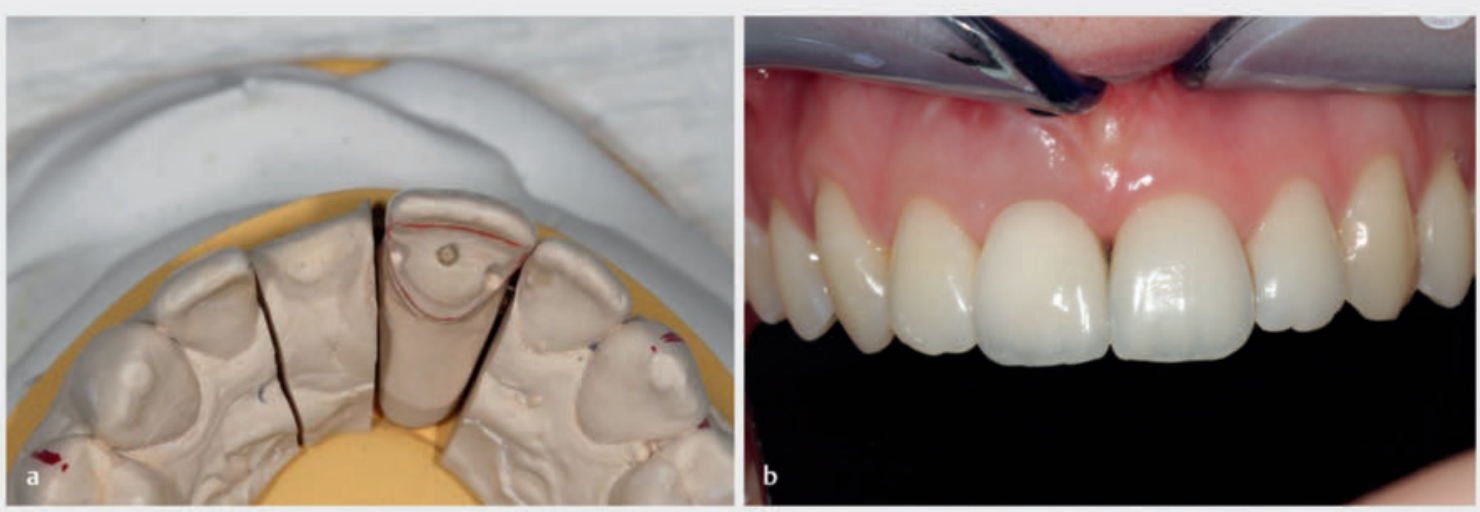

- Abb.11 Einflügelige Metallkeramik-Adhäsivbrücke zum Ersatz des Zahnes 11.

a Präparationsdesign.

b Frontalansicht 2 Wochen nach dem Einsetzen.
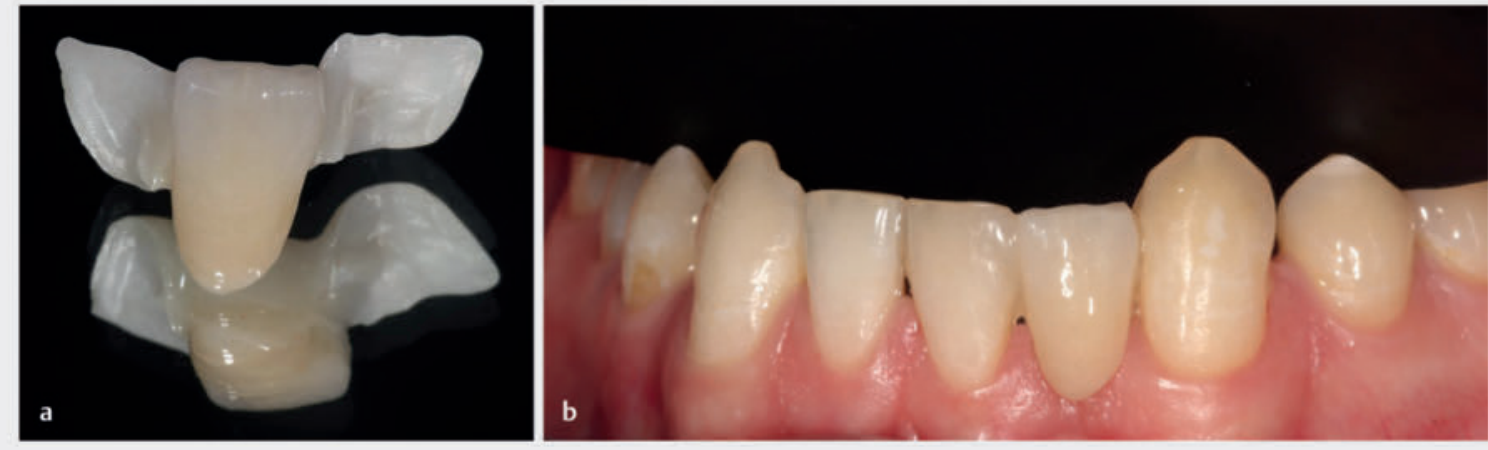

- Abb. 12 Vollkeramische (Zirkoniumdioxid-) Adhäsivbrücke zum Ersatz des unteren linken seitlichen Schneidezahnes. Das retentive Design beruht hier auf einer approximalen Rillenpräparation. Eine Bohrung wurde nicht angelegt, da diese sich im CAD/CAMFräsprozess nicht retentiv umsetzen lässt.

a Detailansicht.

b Klinische Frontalansicht.

\section{Merke}

CoCr-Adhäsivbrücken sollten nach einem Korundstrahlprozess unter Verwendung MDP-haltiger Adhäsivzemente und/oder in Kombination mit MDP-haltigen Primern eingesetzt werden. Alternativ können die Adhäsivflügel tribochemisch silikatisiert und ein Silan aufgetragen werden und anschließend ein beliebiger Adhäsivzement verwendet werden.

\section{Vollkeramische Adhäsivbrücken im Frontzahnbereich}

Der Wunsch einiger Patienten nach zahnfarbenen, metallfreien Versorgungen hat auch vor den Adhäsivbrücken keinen Halt gemacht ( $\triangleright$ Abb. 12).
Das vor allem bei sehr transluzenten Schneidezähnen mögliche „Durchschimmern“ des metallischen Gerüstmaterials soll so vermieden werden. In den 1990er-Jahren wurden die ersten Adhäsivbrücken aus glasinfiltrierten Aluminiumoxidkeramikgerüsten mit glaskeramischer Verblendung des Brückenglieds hergestellt. Nach weiter Verbreitung und Verbesserung haben sich heutzutage aufgrund einfacher Verarbeitung mittels CAD/CAM-Verfahren sowie höherer Bruchlasten Zirkoniumdioxid-basierte Restaurationen durchgesetzt. Ein deutlicher Nachteil von Zirkoniumdioxid ist, dass die Haftung der Adhäsivmaterialien z.T. geringer als bei Glas- oder glasinfiltrierten Oxidkeramiken ausfällt, die Oberflächenkonditionierung kann nicht durch Flusssäureätzung erfolgen [33, 34]. 
Die häufigste Komplikation vollkeramischer Adhäsivbrücken ist Debonding [35]. Löst sich bei 2-flügeligen Brücken nur ein Klebeflügel, spricht man von partiellem Debonding. Bleibt dies unbemerkt, kann Karies die Folge sein [36]. Therapeutisch gesehen muss bei einem partiellen Debonding in der Regel die gesamte Adhäsivbrücke entfernt und erneuert werden. Die zweithäufigste Komplikation vollkeramischer Adhäsivbrücken ist die Fraktur; sowohl die Fraktur als auch ein Debonding wurden bei vollkeramischen Adhäsivbrücken häufiger beobachtet, wenn ein 2-flügeliges Design verwendet wurde [35].

Als 1-flügelige Variante ausgeführt, deuten 10-Jahres-Daten darauf hin, dass die Überlebenswahrscheinlichkeit bei über $90 \%$ liegt, was vergleichbar mit konventionellen Brückenversorgungen und Einzelzahnimplantaten ist. Man sollte aber mit einer aufwendigeren Nachsorge durch Debonding rechnen. Die Patienten sollten idealerweise ausführlich über die Vor- und Nachteile aufgeklärt werden. Der Komplikation des Debondings kann allerdings recht einfach mit Säuberung des Pfeilerzahnes und der Restauration und erneuter Befestigung begegnet werden. Bei der Planung von 1-flügeligen Adhäsivbrücken sollte man aber zwingend berücksichtigen, dass keine isolierten dynamischen Kontakte auf dem Klebeflügel vorhanden sein dürfen. Das Brückenglied darf keine dynamischen Okklusionskontakte aufweisen. Eine Übersichtarbeit aus dem Jahr 2018 stellt fest, dass eine Aussage zur Entscheidung, ob Klebebrücken 1- oder 2-flügelig angefertigt werden sollen, nicht absolut evidenzbasiert getroffen werden kann. Dies begründen die Autoren damit, dass randomisierte Vergleichsstudien fehlen und in den verschiedenen klinischen Studien die Auswahlkriterien für die Patienten unterschiedlich sind [16]. Aufgrund der Schwierigkeit, Zirkoniumdioxid zu verkleben, beschäftigen sich seit jeher Wissenschaftler und Industrie damit, Konditionierungsstrategien zu untersuchen und zu verbessern. In einer eigenen Studie wurde der Einfluss von 6 verschiedenen Oberflächenkonditionierungen und 4 verschiedenen Adhäsivsystemen auf das Zirkoniumdioxidbonding unter Laborbedingungen untersucht. Nach 150 Tagen Wasserlagerung und 37000 Zyklen Thermowechselbelastung überzeugte tribochemisches Silikatisieren, wenn anschließend ein Silan- und MDP-haltiger Primer (Clearfil Ceramic Primer) aufgetragen und ein MDP-haltiger Adhäsivzement (Panavia 21) verwendet wurde [37]. Diese Kombination erwies sich auch klinisch als effektiv bei der Verankerung minimalinvasiver Zirkoniumdioxidbrücken [38]. Daneben wird ein 50- $\mu \mathrm{m}$-Korundstrahlprozess bei moderatem Druck (0,5-2,5 bar) in Kombination mit einem MDP-haltigen Primer/Zement als dazu geeignet angesehen, Zirkoniumdioxid klinisch dauerhaft zu verkleben [39].
Merke
Vollkeramische Adhäsivbrücken aus Zirkoniumdioxid können eine Alternative zu metallkeramischen sein. Neben ästhetischen Vorteilen sollten die Patienten aber über mögliche Komplikationen wie Debonding, Chipping und Frakturen aufgeklärt werden.

\section{Vollkeramische Adhäsivbrücken im Seitenzahnbereich}

Vor allem im Unterkieferseitenzahnbereich sind Metallbasierte Adhäsivbrücken ästhetisch einschränkend, da die Adhäsivflügel die Kauflächen zum Teil bedecken.

Gemäß neuester S3-Leitlinie Vollkeramische Kronen und Brücken kann allerdings für vollkeramische Adhäsivbrücken im Seitenzahnbereich keine evidenzbasierte Empfehlung ausgesprochen werden. Aktuelle Daten aus einer Studie mit 30 Patienten, die mit einer monolithischen Zirkoniumdioxid-Adhäsiv- bzw. Inlaybrücke versorgt wurden, ergaben kurzfristig eine hohe und komplikationsarme Überlebenswahrscheinlichkeit [38]. Die Präparation für vollkeramische Adhäsivbrücken ist ähnlich wie die für die metallkeramische Variante ( $\bullet$ Abb. 13).

Es ist eine leichte Hohlkehlpräparation der oralen und approximalen Zahnflächen anzulegen; der Substanzabtrag sollte mind. 0,5 mm betragen. Die oralen Kauflächenbereiche sollten um mind. 0,7 mm reduziert werden, um die materialspezifische Mindestschickstärke von 0,6 mm zu erreichen. Als retentives Element wird okklusal entlang der Zentralfissur eine Kastenpräparation (1 mm tief, $1 \mathrm{~mm}$ breit) mit einem konischen Feinkorndiamanten angelegt. Bestehende Füllungen sollten defektorientiert in
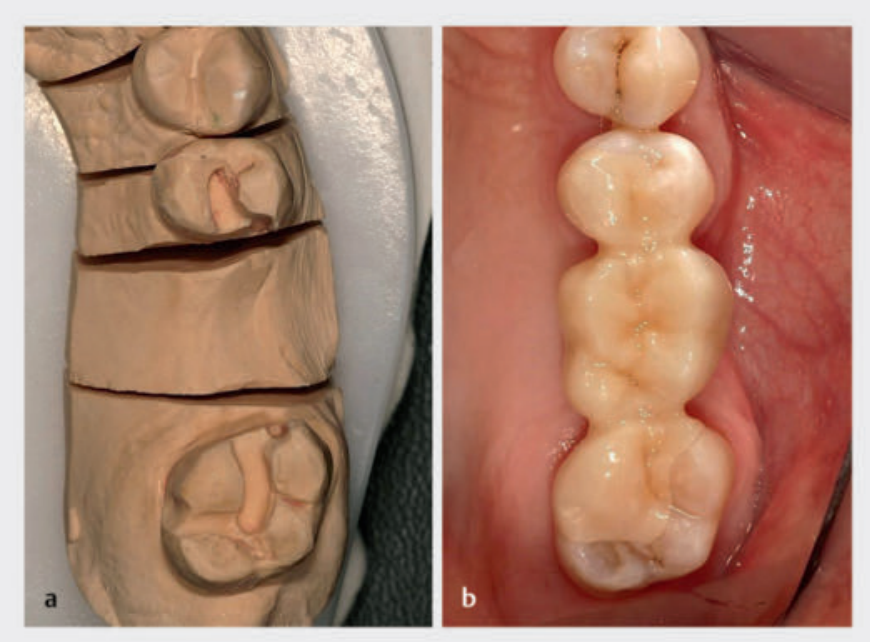

- Abb. 13 Monolithische vollkeramische (Zirkoniumdioxid-) Adhäsivbrücke im Seitenzahnbereich.

a Präparationsdesign.

b Okklusalansicht mit eingesetzter Brücke. 
die Präparation mit einbezogen werden. Abschließend wird approximal an beiden Pfeilerzähne eine Rille $(3 \mathrm{~mm}$ lang) präpariert. Hierbei ist darauf zu achten, dass diese etwas deutlicher ausfällt als bei der Präparation für metallkeramische Adhäsivbrücken, da die Zirkonoxidbrücken im CAD/CAM-Verfahren hergestellt werden (Scanbarkeit/Fräsbarkeit). In der o.g. klinischen Studie hat sich nachfolgendes Klebeprotokoll als erfolgversprechend erwiesen:

1. tribochemische Silikatisierung (Rocotec Pre und Plus),

2. Auftragen eines MDP-haltigen Primers (Clearfil Ceramic Primer),

3. selektive Ätzung (10 s, 37\% Phosphorsäure) der Schmelzbegrenzungen,

4. Auftragen eines MDP-haltigen Ein-Schritt-Haftvermittlers auf die gesamte Präparation (ED-Primer) und

5. MDP-haltiger Adhäsivzement (Panavia 21).

Falls erforderlich eignen sich zur Bearbeitung auch von Zirkoniumdioxid vorgesehene ei- und flammenförmige Feinkorndiamanten zur Ausarbeitung der Zementfuge. Poliert werden kann mit speziellen Zirkoniumdioxidpoliersets, z. B. mit dem 2-stufigen System der Fa. Komet.

\section{Vollkeramische Inlaybrücken im Seitenzahnbereich}

Je nach Defektlage und -ausmaß der Pfeilerzähne ist als Alternative zu Adhäsivbrücken auch die Anfertigung von Inlaybrücken möglich. In einer frühen Studie zu 3-gliedrigen vollkeramischen Inlaybrücken $(n=30)$ konnten allerdings bereits nach einem Jahr zahlreiche fatale Komplikationen beobachtet werden, die fast immer auch zum Versagen der Restauration führten (neben Debonding
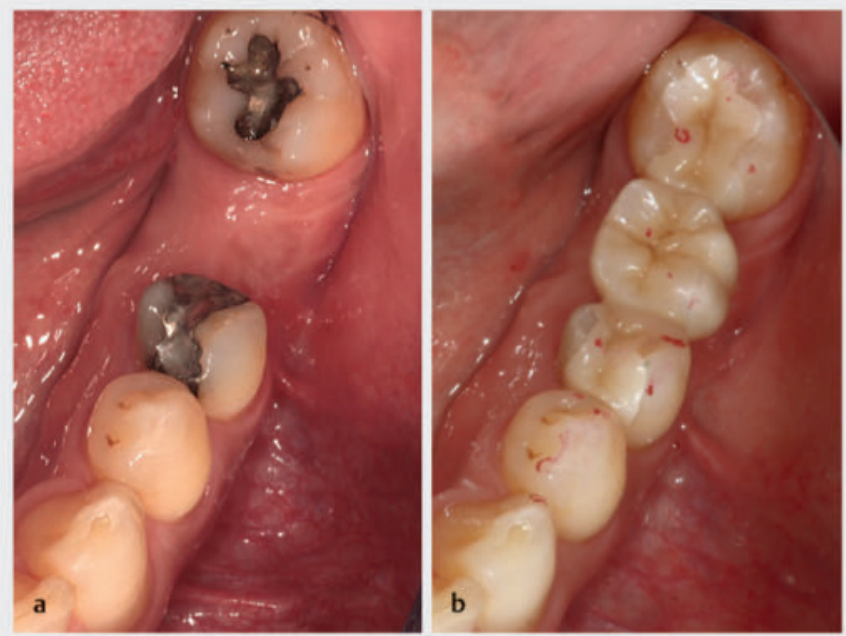

Abb. 14 Monolithische Zirkoniumdioxid-Inlaybrücke mit klassischer Inlaypräparation.

a Ausgangssituation mit bestehenden Amalgamfüllungen.

b Okklusalansicht mit eingesetzter Brücke. auch 3 Gerüstbrüche und 4 Abplatzungen der Verblendkeramik) [46]. Nach 10 Jahren Beobachtungsdauer hatten rund $90 \%$ der Brücken versagt.

Eine Modifikation des Präparationsdesigns mit Vergrößerung der Klebefläche durch Erweiterungen (Flügelchen) nach oral und vestibular führte zu einer verbesserten klinischen Leistungsfähigkeit; nach einer mittleren Beobachtungszeit von 5 Jahren traten bei 3 der 30 Studienbrücken ein Chipping der Verblendkeramik und bei 2 Brücken Retentionsverluste auf [47]. Die 5-Jahres-Überlebensrate betrug 98,5\%.

Da Verblendfrakturen bei Zirkoniumdioxid-basierten Keramiken eine häufige Komplikation darstellen, wurde eine Studie zur klinischen Leistungsfähigkeit monolithischer Inlaybrücken aufgelegt [38]. Nach bisher 1 Jahr liegt die Überlebensrate der Restaurationen mit klassischer Inlaypräparation bei $100 \%$ ( Abb. 14).

\section{Faserverstärkte Kompositbrücken im Frontzahnbereich}

Es ist empfehlenswert, zur Therapieplanung zunächst Situationsmodelle herzustellen. Auf Basis dieser Modelle können die verschiedenen Therapieoptionen mit dem Patienten besprochen werden. Anhand der Modelle kann neben der klinischen Befundung überprüft werden, ob eine Rillenpräparation für den FRC-Flügel notwendig ist. Bei der Anfertigung einer FRC-Brücke sind unterschiedliche Herstellungsverfahren zu beachten ( $\mathbf{A}$ Ab. 15) [40].

\section{Semidirektes Verfahren}

Im semidirekten Verfahren kann die FRC-Brücke nach einer Abformung im Labor oder Chairside hergestellt werden und anschließend intraoral eingegliedert werden. Dies verringert die intraorale Behandlungszeit für den Patienten, der Nachteil besteht jedoch darin, dass der FRC-Strang bereits ausgehärtet ist und durch „Anfrischung“ mit Monomeren aus dem Adhäsiv (Infiltration) wieder reaktiviert werden muss [41]. Diese Art der Reaktivierung durch Infiltration ist jedoch nicht bei allen Fasermaterialien gleichermaßen erfolgreich. Wird dieses Vorgehen gewünscht, sollte (um die Haftkraft der FRC-Brücke zu erhöhen) unbedingt darauf geachtet werden, dass FRC-Stränge mit einer sogenannten Semi-IPN-Matrix verwendet werden [42]. 


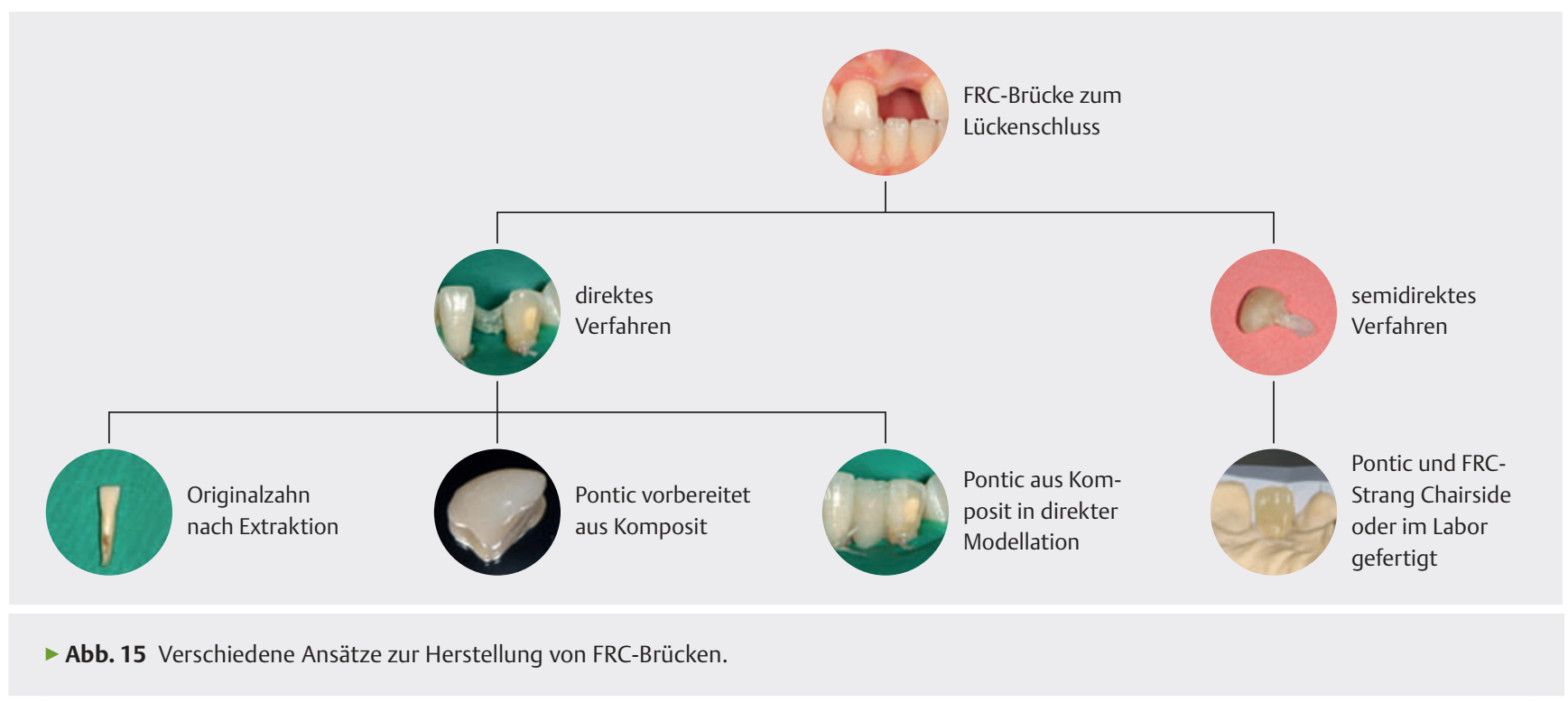

\section{Direktes Verfahren}

Im direkten Verfahren werden hinsichtlich des Pontic-Designs 3 Möglichkeiten unterschieden:

\section{Verwendung eines Originalzahnes als Pontic}

Erscheint nach Extraktion eines Zahnes die Zahnkrone intakt und verwertbar, so kann der Originalzahn zum Pontic umgearbeitet und mit einem frischen unpolymerisierten FRC-Strang direkt intraoral eingeklebt werden. Vorteilhaft ist hier die hohe Haftkraft des Pontics am FRC-Strang aufgrund des Vorhandenseins von natürlichem Schmelz und Dentin.

\section{Extraorale (indirekte) Pontic-Modellation}

Ist der Originalzahn nicht verwendbar, so kann auf dem Situationsmodell im Labor oder chairside ein Pontic aus Komposit modelliert werden, welches dann nach Vorbereitung intraoral mit einem frischen FRCStrang direkt eingeklebt wird. Auch hier sollte geprüft werden, ob aus Platzgründen eine Rille zur Aufnahme des FRC-Strangs in das Pontic präpariert werden muss.

\section{Intraorale (direkte) Pontic-Modellation}

Wird das Pontic direkt intraoral modelliert, so wird zunächst der Faserstrang als Gerüst eingebracht und anschließend wird das Pontic um den FRC-Strang herum modelliert. Hierbei handelt es sich um die zeitaufwendigste Therapieoption.

\section{Gerüstdesign}

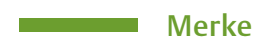

Bei dem Ersatz von einem Zahn sollte aus Stabilitätsgründen mind. ein FRC-Strang als Gerüst verwendet werden. Bei zwei zu ersetzenden Zähnen sollten es mindestens zwei FRC-Stränge sein.

Der Verlauf des FRC-Stranges im Frontzahnbereich sollte sich im inzisalen Bereich des Pontics und des Pfeilerzahnes befinden. Grund hierfür ist, dass die höchsten Peaks der Belastung durch Scherkräfte im inzisalen Bereich entstehen [43]. Hinzu kommt, dass der Faserverlauf im Pontic-Bereich wenn möglich leicht kurvig verlaufen sollte. Eine 1-flügelige Gestaltung von FRC-Brücken im Frontzahnbereich scheint, wie bei laborgefertigten Zirkoniumdioxid-Adhäsivbrücken gezeigt [19], für das Überleben vorteilhaft. Bei geringer Pfeilerwertigkeit kann ein 2-flügeliges Design erwogen werden. Hier ist jedoch zu beachten, dass es bei unterschiedlichen Lockerungsgraden der Pfeilerzähne zum Debonding kommen kann.

\section{Misserfolge und Reparaturmöglichkeiten}

Wie eingangs bereits aufgeführt, sind die häufigsten Komplikationen bei FRC-Brücken Chippingfrakturen, Debonding oder ein kompletter Verlust der Restauration $[29,44,45]$. Aufgrund der Methacrylat-basierten Materialien, die bei FRC-Brücken zum Einsatz kommen, können Defekte wie Debonding, Chippingfrakturen, etc. mit herkömmlichen Kompositen zuverlässig repariert oder FRC-Brücken wiederbefestigt werden. In > Tab. 2 sind die hierfür erforderlichen klinischen Schritte bei der intraoralen Reparatur einer FRC-Brücke mit Komposit aufgeführt. 


\section{FALLBEISPIEL}

\section{Klinisches Beispiel: Herstellung einer FRC-Brücke mit Originalzahn}

Ein Junge (Alter < 15) stellt sich in der Sektion für Präventive und Restaurative Zahnheilkunde unserer Poliklinik für Zahnerhaltungskunde nach Überweisung aus unserer Poliklinik für Kieferorthopädie vor. Der Zahn 21 weist eine Ankylose mit Wurzelresorption nach Frontzahntrauma auf und soll entfernt werden. Ein Lückenschluss durch eine FRC-Brücke mit dem Originalzahn direkt nach Extraktion ist aus ästhetischen und funktionellen Gründen gewünscht ( $>$ Abb. 16a). Als langfristige Therapie zum Lückenschluss ist eine Mesialisierung des Zahnes 22 an Stelle 021 geplant. Beim kieferorthopädischen Lückenschluss soll der Originalzahn schrittweise approximal reduziert werden.

Direkt nach der Extraktion erscheint der Patient und bringt den Originalzahn mit. Da es die okklusalen Verhältnisse erlauben, kann von einer Rillenpräparation am Pfeilerzahn 11 abgesehen werden ( $\bullet$ Abb. 16b-e). Bei der Umarbeitung des Originalzahnes zum Pontic werden zunächst die Wurzel abgetrennt und die Pulpakammer ausgehöhlt ( $\bullet$ Abb. 16f) Anschließend wird der Originalzahn angeätzt, abgespült und mit Luft getrocknet; Primer und Adhäsiv werden aufgetragen ( $\bullet \mathbf{A b b} \mathbf{1 6} \mathbf{g}$ ). Zur Ausformung des Pontics wird Restaurationskomposit auf den Originalzahn unter Beachtung einer eiförmigen Gestaltung der Basalfläche aufgebracht, das Pontic ausgearbeitet und in einem Lichtschutzgefäß gelagert. ( $\vee$ Abb. $\mathbf{1 6} \mathbf{h}$ und i). Vor dem Einkleben des Pontics sollten die Schritte der Adhäsivtechnik wiederholt werden. Zur Bestimmung der Länge des benötigten FRC-Strangs wird die Lückendistanz in Regio 021 ausgemessen und die für den palatinalen Flügel an Zahn 11 benötigte Länge addiert ( $>$ Abb. 16 j). Zunächst wird der Pfeilerzahn 11 angeätzt, abgespült und getrocknet. Anschließend werden Primer und Adhäsiv appliziert. Bei der Befestigung des Originalzahnes empfiehlt es sich, diesen mit einer kleinen Menge Flowkomposit interdental am Pfeilerzahn in seiner korrekten Position zu befestigen, bevor der FRCStrang palatinal aufgelegt wird. Nach erfolgter Befestigung des Pontics mit Flowkomposit wird palatinal auf den Pfeilerzahn und das Pontic eine kleine Menge Flowkomposit aufgetragen, in welches der FRC-Strang platziert und vorsichtig angedrückt wird. Beim Einbringen des FRC-Strangs empfiehlt es sich, diesen schrittweise anzudrücken und an jeder Stelle initial für ca. $3 \mathrm{~s}$ „anzuhärten“. Ist die korrekte Platzierung des Strangs erreicht, so kann dieser mit der Polymerisationslampe vollständig gehärtet werden. Nach Ausarbeitung und Politur erfolgen die Anpassung von Interdentalraumbürstchen sowie die Instruktion des Patienten und dessen Eltern ( Abb. 16k-m).

Bei der Kontrolle nach 2 Monaten stellen sich die Weichteilverhältnisse nach Extraktion des Zahnes 21 unauffällig und reizlos dar. Der Patient reinigt mit der Interdentalraumbürste und die Sondierungstiefen liegen bei $2 \mathrm{~mm}$ ohne Anzeichen auf Blutung nach Sondieren ( $\vee$ Abb. 17a-d).
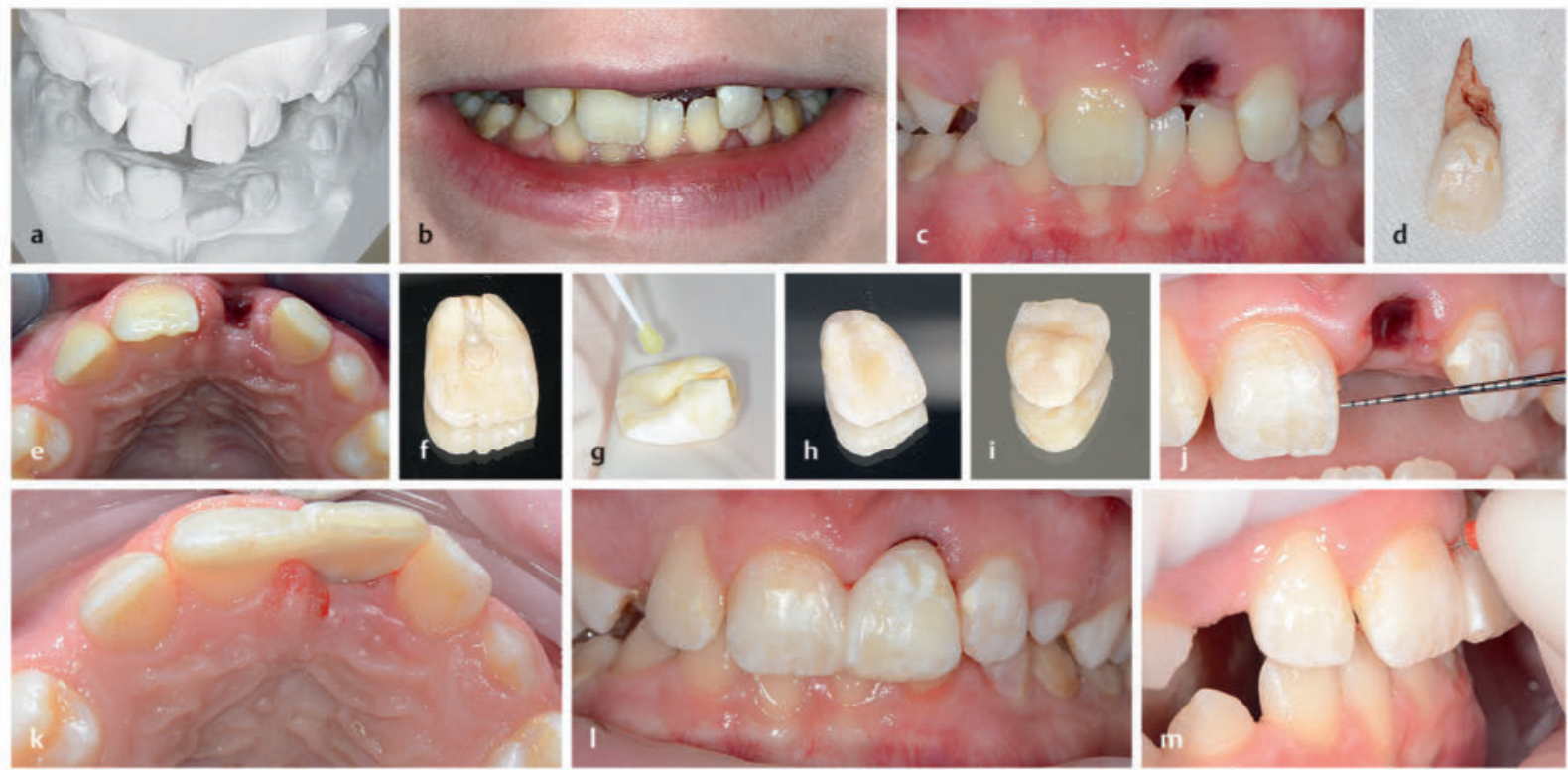

- Abb. 16 Klinisches Beispiel eines jungen männlichen Patienten zur Herstellung einer 1-flügeligen FRC-Brücke 11-021 im direkten Verfahren unter Umarbeitung des Originalzahnes zum Pontic. Das detaillierte Vorgehen ist im Text beschrieben. 

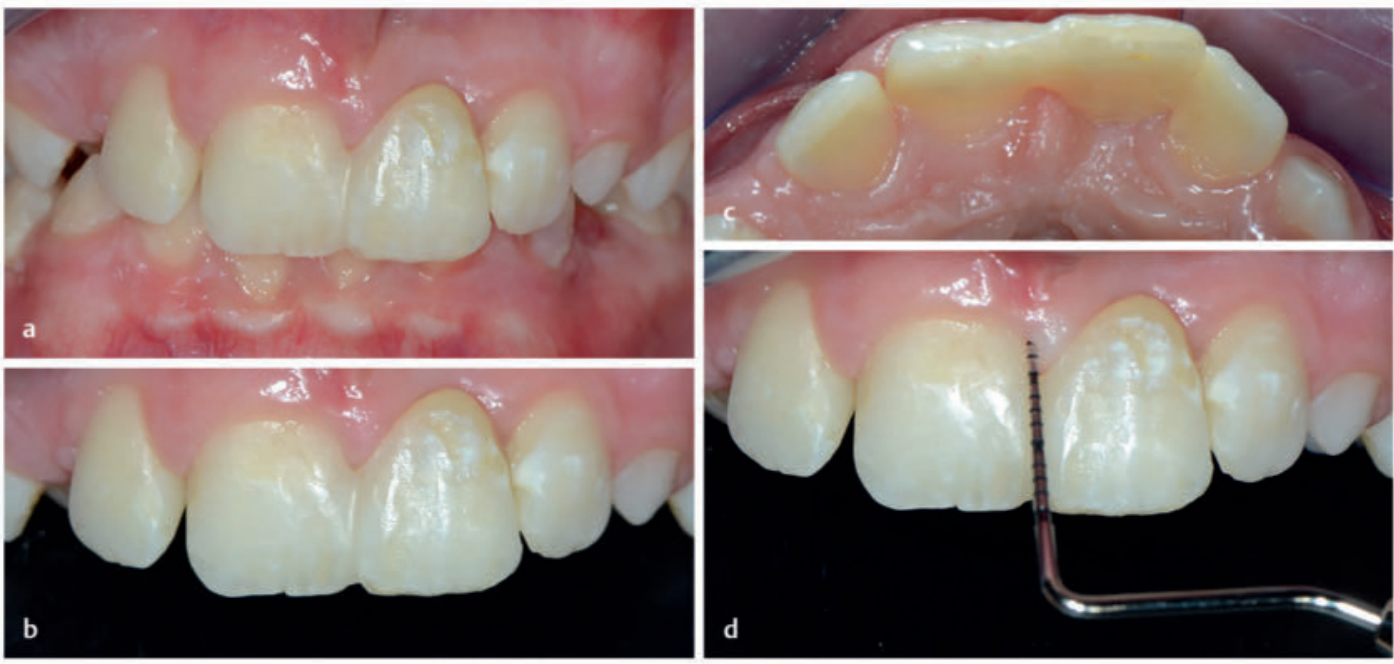

- Abb. 17 Kontrolle nach Eingliederung der FRC-Brücke 11-021 mit dem Originalzahn als Pontic nach 2 Monaten. Die Weichteilverhältnisse nach Extraktion des Zahnes 21 stellen sich unauffällig und reizlos dar. Der Patient reinigt mit einer Interdentalraumbürste und die Sondierungstiefen liegen bei $2 \mathrm{~mm}$ ohne Anzeichen von Blutung nach Sondieren.

- Tab. 2 Erforderliche Schritte bei einer intraoralen Reparatur einer FRC-Brücke mit Komposit.

\begin{tabular}{|l|l|}
\hline Reparaturschritte & empfohlene Zeit \\
\hline Mikrosandstrahlen & abhängig von den Adhärenden \\
\hline $37,5 \%$ Phosphorsäure-Gel & $\begin{array}{l}\text { ca. } 15 \mathrm{~s} \text { Dentin, Wurzeldentin } \\
\text { ca. } 30 \mathrm{~s} \text { Schmelz }\end{array}$ \\
\hline Abspülen und Trocknen & ca. $30 \mathrm{~s}$ und ca. $3 \mathrm{~s}$ (Dentin), sonst länger \\
\hline ggf. Haftvermittler (Silan) & ca. $15 \mathrm{~s}$, Verdunstung abwarten \\
\hline Primer und Adhäsiv & $\begin{array}{l}\text { ca. } 15-20 \text { s, Primer vorsichtig verblasen (Cave: Luftbläser darf keine } \\
\text { Wasser- oder Ölreste ausstoßen) }\end{array}$ \\
\hline Komposit applizieren & - \\
\hline Polymerisation Adhäsivschicht (mind. $\left.800 \mathrm{~mW} / \mathrm{cm}^{2}\right)$ & bis $20-40 \mathrm{~s}$ (nach Herstellerangaben) je nach Leistung der Lampe \\
\hline Polymerisation der Kompositschicht (mind. $800 \mathrm{~mW} / \mathrm{cm}^{2}$ ) & $\begin{array}{l}\text { bis } 20-40 \mathrm{~s} \text { (nach Herstellerangaben) je nach Leistung der Lampe } \\
\text { (Schichtstärke bis } 2 \mathrm{~mm} \text {;e nach Opazität/Transparenz eher längere } \\
\text { Härtungszeiten bevorzugen!) }\end{array}$ \\
\hline
\end{tabular}


Interessenkonflikt

Die Autorinnen/Autoren geben an, dass kein Interessenkonflikt besteht.

\section{Autorinnen/Autoren}

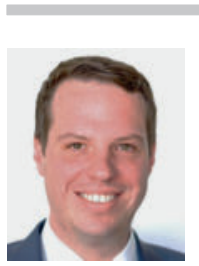

\section{Andreas Zenthöfe}

Prof. (apl.) Dr. med. dent. Studium an der Universität Kiel, danach wissenschaftlicher Mitarbeiter der Poliklinik für Zahnerhaltungskunde der Universität Münster, später wissenschaftlicher Mitarbeiter und seit 2016 Oberarzt in der Poliklinik für Zahnärztliche Prothetik des Universitätsklinikums Heidelberg, Erhalt der Venia Legendi und Ernennung zum Leiter der Sektion Werkstoffkunde und Biomaterialien. Seit 2019 außerplanmäßiger Professor.

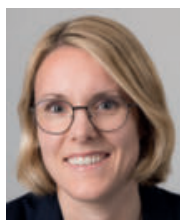

\section{Cornelia Frese}

Prof. (apl.) Dr. med. dent., 2001-2006 Studium an der Ruprecht-Karls-Universität Heidelberg. Danach wissenschaftliche Mitarbeiterin an der Poliklinik für Zahnerhaltungskunde. 2007 Promotion. 2010 Funktionsoberärztin und Erwerb des Zertifikats für Hochschuldidaktik Baden-

Württemberg. 2015 Venia Legendi an der Medizinischen Fakultät der RKU Heidelberg. Seit 2017 apl. Professorin. Leitung der Sektion für Präventive und Restaurative Zahnheilkunde und des Bereichs für Kinder- und Jugendzahnheilkunde der Ruprecht-Karls-Universität Heidelberg.

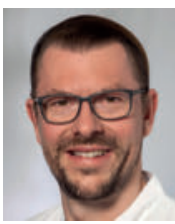

\section{Wolfgang Bömicke}

PD Dr. med. dent., M. Sc. 2002-2007 Studium der Zahnmedizin an der Ruprecht-Karls-Universität (RKU) Heidelberg, danach wissenschaftlicher Mitarbeiter an der Poliklinik für Zahnärztliche Prothetik des Universitätsklinikums Heidelberg (UKHD). 2010-2013 Masterstudium „Zahnärztliche Prothetik“ an der Universität Greifswald. 2011 Promotion. 2015 Ernennung zum Spezialisten für Prothetik nach DGPro. 2017 Ernennung zum Oberarzt der Poliklinik für Zahnärztliche Prothetik des UKHD. 2018 Erlangung der Venia Legendi der Medizinischen Fakultät der RKU Heidelberg.

\section{Korrespondenzadresse}

Prof. (apl.) Dr. med. dent. Cornelia Frese

Poliklinik für Zahnerhaltungskunde

Klinik für Mund-, Zahn- und Kieferkrankheiten

Universitätsklinikum Heidelberg

Im Neuenheimer Feld 400

69120 Heidelberg

Deutschland

cornelia.frese@med.uni-heidelberg.de

\section{Wissenschaftlich verantwortlich}

\section{gemäß Zertifizierungsbestimmungen}

Wissenschaftlich verantwortlich gemäß Zertifizierungsbestimmungen für diesen Beitrag ist Prof. Dr. med. dent. Cornelia Frese, Heidelberg.

\section{Literatur}

[1] Pjetursson BE, Brägger U, Lang NP et al. Comparison of survival and complication rates of tooth-supported fixed dental prostheses (FDPs) and implant-supported FDPs and single crowns (SCs). Clin Oral Implants Res 2007; 18 Suppl 3: 97-113

[2] Kuijs R, van Dalen A, Roeters J et al. The Resin-Bonded Fixed Partial Denture as the First Treatment Consideration to Replace a Missing Tooth. Int J Prosthodont 2016; 29: 337-339

[3] Kern M. Single-retainer resin-bonded fixed dental prostheses as an alternative to orthodontic space closure (and to singletooth implants). Quintessence Int 2018; 49: 789-798

[4] Kullar AS, Miller CS. Are There Contraindications for Placing Dental Implants? Dent Clin North Am 2019; 63: 345-362

[5] Muller F, Salem K, Barbezat C et al. Knowledge and attitude of elderly persons towards dental implants. Gerodontology 2012; 29: e914-e923

[6] Augusti D, Augusti G, Re D. Prosthetic restoration in the single-tooth gap: patient preferences and analysis of the WTP index. Clin Oral Implants Res 2014; 25:1257-1264

[7] Edelhoff D, Sorensen JA. Tooth structure removal associated with various preparation designs for posterior teeth. Int J Periodontics Restorative Dent 2002; 22: 241-249

[8] Schwindling FS, Waldecker M, Rammelsberg P et al. Tooth substance removal for ceramic single crown materials-an in vitro comparison. Clin Oral Investig 2019; 23: 3359-3366

[9] Rochette AL. Attachment of a splint to enamel of lower anterior teeth. J Prosthet Dent 1973; 30: 418-423

[10] Howe DF, Denehy GE. Anterior fixed partial dentures utilizing the acid-etch technique and a cast metal framework. J Prosthet Dent $1977 \cdot 37: 28-31$

[11] Thompson VP, Livaditis G]. Etched casting acid etch composite bonded posterior bridges. Pediatr Dent 1982; 4: 38-43

[12] Behr M, Leibrock A, Stich W et al. Adhesive-fixed partial dentures in anterior and posterior areas. Results of an on-going prospective study begun in 1985. Clin Oral Investig 1998; 2: 31-35

[13] Thoma DS, Sailer I, loannidis A et al. A systematic review of the survival and complication rates of resin-bonded fixed dental prostheses after a mean observation period of at least 5 years. Clin Oral Implants Res 2017; 28: 1421-1432

[14] Balasubramaniam GR. Predictability of resin bonded bridges a systematic review. Br Dent J 2017; 222: 849-858

[15] Yoshida T, Kurosaki Y, Mine A et al. Fifteen-year survival of resin-bonded vs. full-coverage fixed dental prostheses. J Prosthodont Res 2019; 63: 374-382

[16] Alraheam IA, Ngoc CN, Wiesen CA et al. Five-year success rate of resin-bonded fixed partial dentures: A systematic review. J Esthet Restor Dent 2019; 31: 40-50

[17] Chaar MS, Passia N, Kern M. All-ceramic inlay-retained fixed dental prostheses: An update. Quintessence Int 2015; 46 : 781-788

[18] Pospiech P, Rammelsberg P, Unsöld F. A new design for all-ceramic resin-bonded fixed partial dentures. Quintessence Int 1996; $27: 753-758$

[19] Kern M, Passia N, Sasse M et al. Ten-year outcome of zirconia ceramic cantilever resin-bonded fixed dental prostheses and the influence of the reasons for missing incisors. ] Dent 2017; 65: 51-55

[20] Abou Tara M, Eschbach S, Wolfart S et al. Zirconia ceramic inlay-retained fixed dental prostheses - first clinical results with a new design. J Dent 2011; 39: 208-211 
[21] Staehle HJ. Lowtech-Dentistry - Bewährte und neue frugale Interventionen in der Zahnmedizin. Zahnärztl Mitt 2019; 109: 1081-1096

[22] Staehle HJ. Frugale Innovationen in der Zahnmedizin. Zahnmedizin up2date 2016; 10: 197-198

[23] Freilich MA. Fiber-reinforced Composites in clinical Dentistry. Chicago: Quintessence Publishing Co; 2001

[24] Proctor BA. Composite Material I: Fibre reinforced composite Materials: An introductory Review. Lathom, Ormskirk, Lancashire: Pilkington Research and Development Laboratories; 1976: 63-75

[25] Smith DC. Recent developments and prospects in dental polymers J Prosthet Dent 1962; 12: 1066-1078

[26] Frese C, Staehle H], Wolff D. Faserverstärkte Komposite - Materialspezifische Eigenschaften und klinische Einsatzmöglichkeiten. wissen kompakt 2015; 9: 179-188

[27] Vallittu PK, Shinya A, Baraba A et al. Fiber-reinforced composites in fixed prosthodontics-Quo vadis? Dent Mater 2017; 33: 877-879

[28] Frese C. Glasfaserverstärkte Restaurationen. Zahnärztl Mitt 2020; 110: 48-56

[29] Wolff D, Wohlrab T, Saure D et al. Fiber-reinforced composite fixed dental prostheses: A 4-year prospective clinical trial evaluating survival, quality, and effects on surrounding periodontal tissues. J Prosthet Dent 2018; 119: 47-52

[30] Boemicke W, Kappel S, Stober T et al. Clinical comparison of metal ceramic resin-bonded fixed dental prostheses with a conventional and a mixed retainer design. J Prosthet Dent 2014; 112: 472-480

[31] Frese C, Staehle HJ. Wie invasiv ist minimalinvasiv? - Management von Einzelzahnlücken aus konservierender Sicht. Der Freie Zahnarzt 2018; 4: 252-259

[32] Audenino G, Giannella G, Morello GM et al. Resin-bonded fixed partial dentures: ten-year follow-up. Int J Prosthodont 2006; 19: $22-23$

[33] Kern M, Wegner SM. Bonding to zirconia ceramic: adhesion methods and their durability. Dent Mater 1998; 14: 64-71

[34] Tzanakakis EG, Tzoutzas IG, Koidis PT. Is there a potential for durable adhesion to zirconia restorations? A systematic review. J Prosthet Dent 2016; 115: 9-19

[35] Chen J, Cai H, Ren X et al. A Systematic Review of the Survival and Complication Rates of All-Ceramic Resin-Bonded Fixed Dental Prostheses. J Prosthodont 2018; 27: 535-543

[36] Lang NP, Pjetursson BE, Tan K et al. A systematic review of the survival and complication rates of fixed partial dentures (FPDs) after an observation period of at least 5 years. II. Combined tooth-implant-supported FPDs. Clin Oral Implants Res 2004; 15: 643-653
[37] Bömicke W, Schürz A, Krisam J et al. Durability of Resin-Zirconia Bonds Produced Using Methods Available in Dental Practice. J Adhes Dent 2016; 18: 17-27

[38] Bömicke W, Rathmann F, Pilz M et al. Clinical Performance of Posterior Inlay-Retained and Wing-Retained Monolithic Zirconia Resin-Bonded Fixed Partial Dentures: Stage One Results of a Randomized Controlled Trial. J Prosthodont 2020. doi:10.1111/jopr.13258

[39] Kern M. Bonding to oxide ceramics-laboratory testing versus clinical outcome. Dent Mater 2015; 31: 8-14

[40] Wolff D, Schach C, Kraus T. Faserverstärkte Kompositbrücken. Zahnmedizin up2date 2009; 2: 55-78

[41] Wolff D, Geiger S, Ding P et al. Analysis of the interdiffusion of resin monomers into pre-polymerized fiber-reinforced composites. Dent Mater 2012; 28: 541-547

[42] Frese C, Decker C, Rebholz J et al. Original and repair bond strength of fiber-reinforced composites in vitro. Dent Mater 2014; 30: 456-462

[43] Yokoyama D, Shinya A, Lassila LV et al. Framework design of an anterior fiber-reinforced hybrid composite fixed partial denture: a 3D finite element study. Int J Prosthodont 2009; 22: 405-412

[44] Frese C, Schiller P, Staehle HJ et al. Fiber-reinforced composite fixed dental prostheses in the anterior area: a 4.5-year followup. J Prosthet Dent 2014; 112: 143-149

[45] Wolff D, Schach C, Kraus T et al. Fiber-reinforced composite fixed dental prostheses: a retrospective clinical examination. J Adhes Dent 2011; 13: 187-194

[46] Rathmann F, Bömicke W, Rammelsberg P et al. Veneered zirconia inlay-retained fixed dental prostheses: 10-Year results from a prospective clinical study. J Dent 2017; 64: 68-72

[47] Chaar MS, Kern M. Five-year clinical outcome of posterior zirconia ceramic inlay-retained FDPs with a modified design. J Dent 2015; 43: 1411-1415

\section{Bibliografie}

Zahnmedizin up2date 2020; 14: 467-483

DOI 10.1055/a-1307-7178

ISSN $1865-0457$

(C) 2020. Thieme. All rights reserved. Georg Thieme Verlag KG, Rüdigerstraße 14, 70469 Stuttgart, Germany 


\section{Punkte sammeln auf CMIE. thiemede}

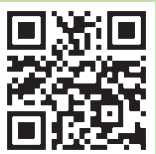

Diese Fortbildungseinheit ist in der Regel 12 Monate online für die Teilnahme verfügbar.

Den genauen Einsendeschluss finden Sie unter https://cme.thieme.de.

Sollten Sie Fragen zur Online-Teilnahme haben, finden Sie unter https://cme.thieme.de/hilfe

eine ausführliche Anleitung. Wir wünschen viel Erfolg beim Beantworten

der Fragen!

Unter https://eref.thieme.de/CXG2RHZ oder über den QR-Code kommen Sie direkt zur Startseite des Wissenstests.

\section{Frage 1}

In welchem Bereich der Zahnheilkunde wurden die ersten faserverstärkten Komposite verwendet?
A Verblockung von Zähnen
B Bulk-Fill-Material
C Verstärkung von Prothesenbasen
D Wurzelkanalstifte
E Knochenersatzmaterial

\section{Frage 2}

In FRC-Materialien können die Fasern auf unterschiedliche Weise angeordnet sein. Wie ist die Faseranordnung in einem FRCStrang, der für eine Adhäsivbrücke verwendet wird?
A unidirektional
B orthogonal
C bidirektional
D zufällig
$\mathrm{E}$ intersektional

\section{Frage 3}

Wo sollte das FRC-Gerüst bei der Herstellung einer FRC-Brücke im Frontzahnbereich platziert werden?

A möglichst weit medial

B möglichst weit inzisal

C möglichst weit zervikal

D möglichst weit bukkal

E möglichst weit approximal

\section{Frage 4}

FRC-Brücken weisen trotz materialspezifischer Limitationen Vorteile auf, die den Einsatz dieser Versorgungsart in gewissen Fällen vorteilhaft machen und rechtfertigen. Besonders hervorzuheben ist hier das...
A „Dynamic Prevention Concept“.
B „Dynamic Restoration Concept“.
C „Dynamic Operation Concept“.
D „Dynamic Preparation Concept“.
E „Dynamic Treatment Concept“.

\section{Frage 5}

Welche klinische Modalität stellt eine Indikation für die Lückenversorgung mittels Adhäsivbrücke dar?

A umfangreiche Füllungen der potenziellen Pfeiler

B Form- oder Stellungskorrektur der Pfeilerzähne erforderlich

C Patient weist ausschließlich Tagbruxismus auf.

D C1-Karies an einer oralen Zahnfläche bei ansonsten kariesund füllungsfreien Nachbarzähnen

E Der Patient weist eine dauerhaft eingeschränkte Mundhygiene bei schlechter Compliance auf.

\section{Frage 6}

Welcher der nachfolgenden Behandlungsschritte im Rahmen der Therapie mittels Adhäsivbrücke trifft nicht zu?

A Zervikaler Abschluss der Präparation wird als dezente Stufenpräparation ausgeführt.

B Bei Adhäsivbrücken im Frontzahnbereich werden als retentive Elemente approximale Rillen und eine Pinbohrung im Bereich des Tuberculums angelegt.

C Bei der Präparation für 1-flügelige Adhäsivbrücken sollte eine 2. Retentionsrille in dem der Lücke abgewandten Approximalraum angelegt werden.

D Die Farbbestimmung sollte im Idealfall erst bei der Gerüstanprobe erfolgen.

E Adhäsivbrücken können mit MDP-haltigen Adhäsivzementen eingesetzt werden.

- Weitere Fragen auf der folgenden Seite... 


\title{
Punkte sammeln auf CME. thieme.de
}

\author{
Fortsetzung...
}

\section{Frage 7}

Welche Aussage zu vollkeramischen Adhäsivbrücken im Frontzahnbereich trifft zu?

A Zu vollkeramischen Adhäsivbrücken liegen noch keine klinischen Daten vor.

B Bei der 1-flügeligen Variante sollte der Patient über ein mögliches spontanes Lösen der Brücke und damit verbundene Verschluckungsgefahr aufgeklärt werden.

C Adhäsivbrücken im Frontzahnbereich sollten aufgrund der vorteilhaften ästhetischen Eigenschaften bevorzugt aus Lithiumdisilikatkeramik hergestellt werden.

D Zirkoniumdioxid ist der Werkstoff der Wahl für Frontzahnadhäsivbrücken, da sich mit Flusssäureätzung eine sichere und dauerhafte Befestigung erreichen lässt.

E Der Substanzabtrag für metallkeramische und vollkeramische Adhäsivbrücken liegt bei ca. $1 \mathrm{~mm}$.

\section{Frage 8}

Welche Antwort ist hinsichtlich der Prognose von Adhäsivbrücken im Frontzahnbereich falsch?

A Bei Realisierung des retentiven Präparationsdesigns zeigen metallkeramische Adhäsivbrücken mit konventionellen Brücken vergleichbare Überlebenswahrscheinlichkeiten.

B Korrekt ausgeführte 1-flügelige vollkeramische Adhäsivbrücken zeigen Überlebenswahrscheinlichkeiten von über $90 \%$ nach 10 Jahren.

C Die häufigste Komplikation 1-flügeliger vollkeramischer Adhäsivbrücken ist Debonding.

D 2-flügelige Adhäsivbrücken können bei unbemerktem Lösen eines Klebeflügels zu Karies am entsprechenden Pfeilerzahn führen.

E Die häufigste biologische Komplikation bei Adhäsivbrücken ist Vitalitätsverlust.

\section{Frage 9}

Was gilt für vollkeramische Adhäsiv- bzw. Inlaybrücken im Seitenzahnbereich?

A Durch Modifikationen wie zusätzliche Retentionselemente oder die Vergrößerung der Klebefläche konnte die klinische Leistungsfähigkeit verbessert werden.

B Vollkeramische Inlaybrücken sind aufgrund ihrer guten und langfristigen klinischen Leistungsfähigkeit Adhäsivbrücken vorzuziehen.

C Die monolithische Ausführung von Adhäsivbrücken scheint zu mehr Komplikationen wie z. B. Chipping zu führen.

D Auf eine retentive Präparation für Adhäsivbrücken kann aufgrund der größeren Klebeflächen verzichtet werden.

E Inlaybrücken sind die Behandlungsoption der 1. Wahl, falls eine Implantation nicht möglich ist.

\section{Frage 10}

Welche Universität war Namensgeber für das häufig für Adhäsivbrücken gebrauchte Synonym (... -Brücke)?
A Universität Toronto
B Universität Kiel
C Universität Heidelberg
D Universität Maryland
E Universität Rochette 